\title{
Polygenic Risk Score in African populations: progress and
}

\section{challenges [version 1; peer review: 2 approved with}

\section{reservations]}

\author{
Yagoub Adam (iD1* ${ }^{*}$, Suraju Sadeeq ${ }^{2,3^{*}}$, Judit Kumuthini4, Olabode Ajayi4, \\ Gordon Wells ${ }^{4}$, Rotimi Solomon 1,3,5, Olubanke Ogunlana (Did,3,5, \\ Emmanuel Adetiba3,6,7, Emeka Iweala3,5, Benedikt Brors ${ }^{8,9}$, Ezekiel Adebiyi (iD1-3,9 \\ ${ }^{1}$ Covenant University Bioinformatics Research (CUBRe), Covenant University, Ota, Ogun State, 112212, Nigeria \\ ${ }^{2}$ Dept Computer \& Information Sciences, Covenant University, Ota, Ogun State, 112212, Nigeria \\ ${ }^{3}$ Covenant Applied Informatics and Communication Africa Centre of Excellence (CApIC-ACE), Covenant University, Ota, Ogun State, \\ 112212, Nigeria \\ ${ }^{4}$ Centre for Proteomic and Genomic Research, Cape Town, Western Cape, South Africa \\ ${ }^{5}$ Dept of Biochemistry, Covenant University, Ota, Ogun State, 112212, Nigeria \\ ${ }^{6}$ Dept of Electrical \& Information Engineering (EIE), Covenant University, Ota, Ogun State, 112212, Nigeria \\ ${ }^{7}$ HRA, Institute for Systems Science, Durban University of Technology, Durban, South Africa \\ ${ }^{8}$ German Cancer Consortium (DKTK), Heidelberg, Germany \\ ${ }^{9}$ Applied Bioinformatics Division, German Cancer Research Center (DKFZ), Heidelberg, 69120, Germany \\ * Equal contributors
}

V1 First published: 14 Feb 2022, 11:175

https://doi.org/10.12688/f1000research.76218.1

Latest published: 11 Apr 2023, 11:175

https://doi.org/10.12688/f1000research.76218.2

\section{Abstract}

Polygenic Risk Score (PRS) analysis is a method that predicts the genetic risk of an individual towards targeted traits. Even when there are no significant markers, it gives evidence of a genetic effect beyond the results of Genome-Wide Association Studies (GWAS). Moreover, it selects single nucleotide polymorphisms (SNPs) that contribute to the disease with low effect size making it more precise at individual level risk prediction. PRS analysis addresses the shortfall of GWAS by taking into account the SNPs/alleles with low effect size but play an indispensable role to the observed phenotypic/trait variance. PRS analysis has applications that investigate the genetic basis of several traits, which includes rare diseases. However, the accuracy of PRS analysis depends on the genomic data of the underlying population. For instance, several studies show that obtaining higher prediction power of PRS analysis is challenging for non-Europeans. In this manuscript, we review the conventional PRS methods and their application to sub-Saharan African communities. We conclude that lack of sufficient GWAS data and tools is the limiting factor of applying PRS analysis to sub-Saharan populations. We recommend developing Africa-specific PRS methods and tools for estimating and

Open Peer Review

Approval Status? ?

1 2

version 2

(revision)

11 Apr 2023

version 1

14 Feb 2022

1. Bingxin Zhao, Purdue University, West Lafayette, USA

2. Cathryn M. Lewis ID), King's College London, London, UK

Any reports and responses or comments on the article can be found at the end of the article. 
analyzing African population data for clinical evaluation of PRSs of interest and predicting rare diseases.

\section{Keywords}

Prediction medicine, GWAS, post-GWAS, PRS analysis, Africa

population

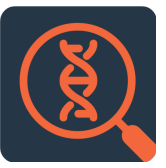

This article is included in the Genomics and

Genetics gateway.

Corresponding author: Ezekiel Adebiyi (ezekiel.adebiyi@covenantuniversity.edu.ng)

Author roles: Adam Y: Conceptualization, Data Curation, Formal Analysis, Investigation, Methodology, Project Administration, Resources, Software, Validation, Visualization, Writing - Original Draft Preparation, Writing - Review \& Editing; Sadeeq S: Conceptualization, Formal Analysis, Visualization, Writing - Original Draft Preparation, Writing - Review \& Editing; Kumuthini J: Conceptualization, Writing - Original Draft Preparation, Writing - Review \& Editing; Ajayi O: Conceptualization, Writing - Original Draft Preparation, Writing - Review \& Editing; Wells G: Writing - Review \& Editing; Solomon R: Conceptualization, Writing - Original Draft Preparation, Writing - Review \& Editing; Ogunlana O: Conceptualization, Writing - Original Draft Preparation, Writing - Review \& Editing; Adetiba E: Conceptualization, Writing - Original Draft Preparation, Writing - Review \& Editing; Iweala E: Conceptualization, Writing - Original Draft Preparation, Writing - Review \& Editing; Brors B: Conceptualization, Writing - Original Draft Preparation, Writing - Review \& Editing; Adebiyi E: Conceptualization, Funding Acquisition, Methodology, Resources, Supervision, Writing - Original Draft Preparation, Writing - Review \& Editing

Competing interests: No competing interests were disclosed.

Grant information: Research reported in this publication is supported by the National Human Genome Research Institute (NHGRI), Office Of The Director, National Institutes Of Health (OD) under award numbers U24HG006941 and U2RTW010679. Also research reported in this publication is supported by the World Bank funding for the ACE Impact projects. The content is solely the responsibility of the authors and does not necessarily represent the official views of the National Institutes of Health and the World Bank. Our special thanks to Kalyani Dhusia for her editorial assistance.

The funders had no role in study design, data collection and analysis, decision to publish, or preparation of the manuscript.

Copyright: ( $) 2022$ Adam Y et al. This is an open access article distributed under the terms of the Creative Commons Attribution License, which permits unrestricted use, distribution, and reproduction in any medium, provided the original work is properly cited.

How to cite this article: Adam Y, Sadeeq S, Kumuthini J et al. Polygenic Risk Score in African populations: progress and challenges [version 1; peer review: 2 approved with reservations] F1000Research 2022, 11:175 https://doi.org/10.12688/f1000research.76218.1

First published: 14 Feb 2022, 11:175 https://doi.org/10.12688/f1000research.76218.1 


\section{Introduction}

Genome-Wide Association Studies (GWAS) can be used successfully to identify associations between hundreds of genomic variations with complex genetic traits. ${ }^{1}$ In general, GWAS report single nucleotides polymorphisms (SNPs) as statistically significant genomic variations associated with the trait of interest when their $p$-values are smaller than a cutoff value of $5 \mathrm{e}-09$ in the African population. ${ }^{2}$ This cutoff value statistically depends on the number of SNPs analyzed. ${ }^{2}$ The $^{2}$ statistically significant SNPs reported by GWAS are used to understand the biomolecular mechanisms of many phenotypic traits including various human diseases. Due to the statistical threshold, GWAS might fail to detect SNPs that are associated with low or moderate risks. ${ }^{3,4}$ The limitation of filtering variants associated with low disease risk increases the GWAS false-negative rate. Also, conventional GWAS can not be used to integrate the polygenic nature of many complex traits. ${ }^{5}$ Therefore, several post-GWAS approaches have been introduced to overcome the above mentioned pitfalls. ${ }^{6,7}$ Due to privacy issues, such as access to the individual level of GWAS data sets, most postGWAS approaches require only GWAS summary statistics. Some public resources for GWAS summary statistics include: the GWAS Catalog, ${ }^{8}$ GWAS Central, ${ }^{9}$ and the $\mathrm{dbGaP}$ database. ${ }^{10,11}$ A distinct approach of performing a postGWAS analysis is known as Polygenic Risk Score (PRS) analysis. The PRS methods map genotype data from a GWAS summary into a single variable used to estimate an individual-level risk score for the phenotypic trait. PRS analysis is used to predict an individual heritability by incorporating all selected SNPs. ${ }^{12}$ However, it is important to consider that not all existing genomics technologies have the capabilities to capture the informative variants among trans-ethnic populations. Nevertheless, obtaining a precise PRS value from case-control studies can be used in personalized medicine. Challenges still exist when translating PRS values from clinical validity to clinical utility. ${ }^{13}$ To successfully perform conventional PRS analysis, two distinct GWAS summaries are required. The first data set (training sample) is used to select the SNPs for PRS analysis and the second data set (from the discovery sample) is used to evaluate the predicted value of PRS methods. The following traditional PRS approaches are discussed in this review: (i) weighted methods that consider the effect sizes derived from GWAS result; (ii) unweighted methods that consider the single marker analysis; (iii) shrinkage methods that consider multivariate analysis. This review focuses on the tools and methods that perform PRS analysis and their applications in understanding the predictive power of PRS analysis. The reviewed PRS tools are chosen based on the following criteria:

1. The approach must perform PRS analysis based on "base" (GWAS) data (summary statistics) and "target" data set (genotypes and phenotypes in each of the target data set),

2. The approach may involve linkage disequilibrium pruning, and

3. The method or approach should be readily available as a tool or package so that it can be executed on any data set.

Besides reviewing PRS methods, we aim to investigate the application of PRS analysis in the African population. It is worth mentioning that the term "African population" covers all those whose ancestors are Africans (including Africans in diaspora). Nevertheless, in this manuscript, the focus is on sub-Saharan Africa. It is important to note that when we searched PubMed for PRS publications on August 21,2021, the query reported 194,203 hits in total (see Figure 1 and text Box 1 for the query terms). For this review, we included articles based on their underlying PRS methods.

\section{Classification of PRS methods}

The different conventional approaches under the umbrella of PRS analysis are presented in Figure 2 and Table 1 . We can categorize PRS methods into two; Bayesian-based and non-Bayesian methods. PRS methods can also be classified using their usage of linkage disequilibrium (LD): PRS methods that incorporate LD and PRS methods which apply LD pruning. To ease the understanding of their underlying algorithms, we grouped the PRS analysis approaches into four (see Table 2). Those with;

1. Clumping with thresholding $(\mathrm{C}+\mathrm{T})$

2. $\quad$-value thresholding

3. Penalized regression

4. Bayesian shrinkage 


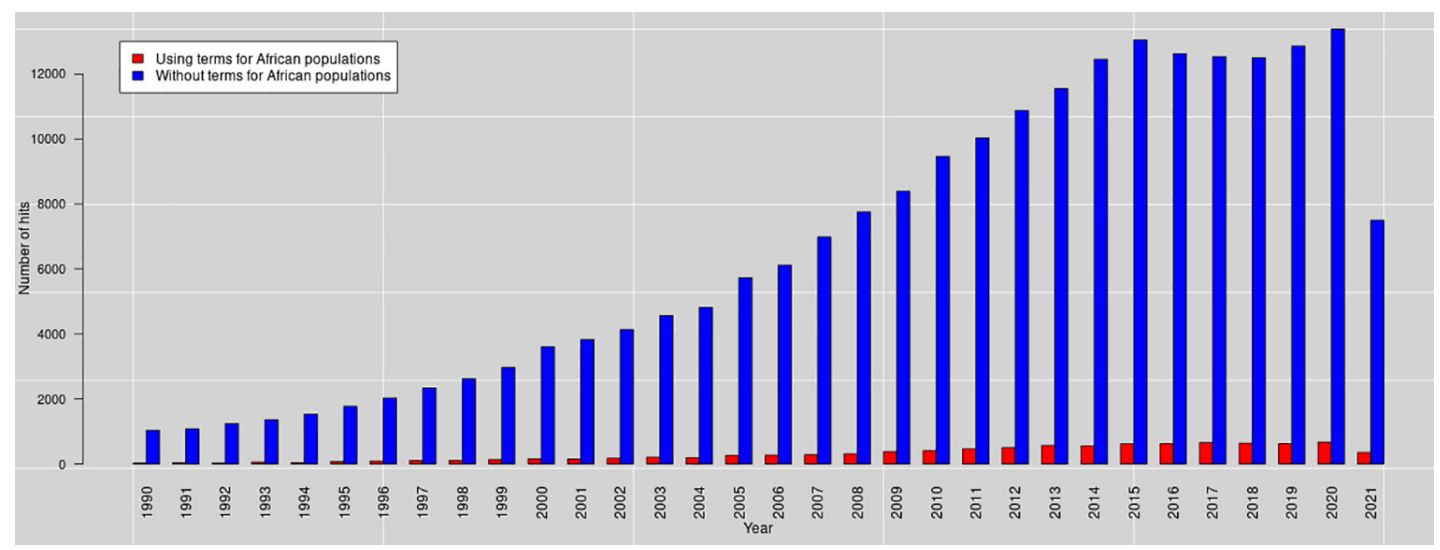

Figure 1. The number of PubMed hits per year (1990-2021) was obtained on August 21, 2021, using query terms for PRS and African populations.

\section{Box 1. Pubmed query terms.}

We used the following terms for querying Pubmed:

((('Polygenic Risk score') OR ((Polygenic) AND (PRS)) OR ('Polygenic score') OR ((Polygenic) AND (PGS)) OR ('Genetic Risk Score') OR (('Genetic Risk') AND (GRS)))

AND

((African) OR (Africa) OR ((Yoruba) AND (YRI)) OR ((Luhya) AND (LWK)) OR ((Mandinka) AND (MAG)) OR ((Mende) AND (MSL)) OR ((Esan) AND (ESN))))

- For PRS terms (in blue color), we included the terms for Genetic Risk Score as some articles used them to refer to PRS.

- For African populations (in red color), we included terms for Africans tribes based on 1000 genomes.

Refer to, ${ }^{14}$ for the query syntax.

PRS methods that incorporate LD

In practice, When the markers are LD pruned, the prediction accuracy of PRS analysis tends to improve. Thus, the absence of LD information limits the predictive accuracy of PRS analysis. ${ }^{15}$ For instance, the method of LD pruning and $p$-value thresholding $(\mathrm{P}+\mathrm{T})$ is commonly used, in the presence of LD patterns to improve the PRS prediction accuracy. ${ }^{16}$ For instance, LDPred (2.0.1) is a Bayesian approach that applies LD information in the presence of LD patterns. From this approach, the posterior mean effects of LD linked loci may be calculated analytically using a Gaussian infinitesimal prior, a non-infinitesimal model, in which only a portion of the markers is causative is perhaps a more realistic prior for effect sizes. For this reason, the following Gaussian mixture prior is considered:

$$
\beta \sim i i d\left\{\begin{array}{ll}
N\left(0, \frac{h_{g}^{n}}{M_{p}}\right) & \text { with probabilityp } \\
0 & \text { with probability }(1-\mathrm{p})
\end{array},\right.
$$

where $p$ refers to the marker's probability as the proportion of causal marker based on the Gaussian distribution. Similarly, the posterior mean in this model can be estimated using the equation below:

$$
E\left(\frac{\beta_{i}}{\widetilde{\beta}^{l}}, D\right) \approx\left(\frac{M}{N h_{g}^{2}} I+D_{i}\right)^{-1} \widetilde{\beta}^{l},
$$

The LD matrix within the LD region is denoted by $D_{i}$ and the estimated effects within the target region are represented by $\widetilde{\beta}$, which is estimated using the least-squares method. The approximation assumes that the heritability explained by the region is small and LD with SNPs outside of the region is negligible. 


\section{Workflow in Polygenic Risk Score Calculation and Analysis}

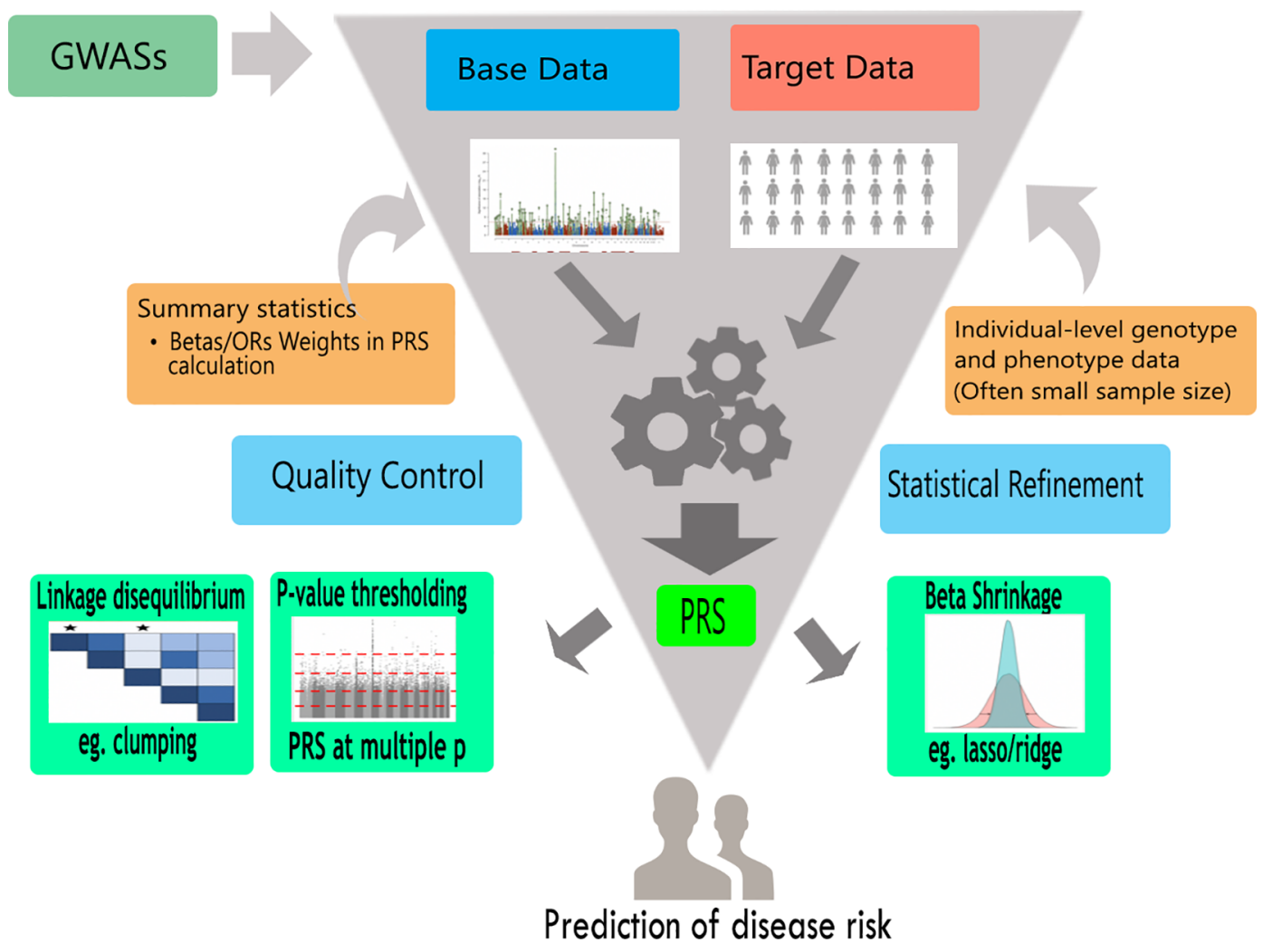

Figure 2. A general PRS analysis workflow. This is a typical polygenic risk score analysis workflow showing base data, target data and encapsulating different approaches. Using genotype and phenotype data,individual-level or summary statistics, approaches such as lasso/ridge regression, clumping and $p$-value thresholding can be employed to increase the predictive accuracy of PRS analysis. Furthermore, the results may be used to predict health or disease risk as well as give information for appropriate therapeutic approaches.

\section{PRS methods that apply LD pruning}

These PRS methods are non-Bayesian approaches that apply informed LD pruning (LD clumping) in PRS computation (Figure 2). Generally, they are known as pruning and thresholding $(\mathrm{P}+\mathrm{T})$ methods. We may apply $p$-value thresholding, for example, with a univariate regression coefficient $\left(r^{2}\right)$ and a threshold of 0.2 . To achieve prediction accuracy in the validation data, we would ensure that the $p$-value thresholding method is optimized across a grid. LD pruning, in which the less significant marker is pruned first, may result in more accurate predictions than random marker pruning. For the $p$ value threshold selection, researchers should include only SNPs that are statistically significant in GWAS. This technique essentially shrinks all omitted SNPs to zero estimates and does not perform shrinkage on the effect size estimates of the included SNPs. The optimal $p$-value threshold is a priori unknown and the targeted phenotype is assessed for the chosen threshold, which is why PRS is commonly computed over several thresholds. This technique can be interpreted as a variable selection process that essentially executes the GWAS $p$-value forward selection based on the size of the increment in the $p$-value thresholds.

\section{Bayesian approach in PRS analysis}

Bayesian techniques have been successfully applied to model pre-existing genetic architecture with a prior that accounts for the range of effect sizes and thus increases polygenic score accuracy. The Bayesian statistical approach computes a refined posterior distribution from prior probability distributions using available data such as functional annotations. It shrinks marker effects by using LD information from a reference panel. ${ }^{17}$ The key benefit of Bayesian-based PRS analysis is its ability to enhance PRS prediction accuracy from summary statistics by taking LD among markers into consideration. ${ }^{18}$ Bayesian approaches in PRS explicitly model pre-existing genetic architecture that accounts for the distribution of effect sizes. These approaches allow the introduction of prior probability that improves the prediction accuracy of a polygenic score. 
Table 1. Summary of polygenic risk score tools.

\begin{tabular}{|c|c|c|c|c|}
\hline Tool & Approach & $\begin{array}{l}\text { Computational } \\
\text { platform }\end{array}$ & $\begin{array}{l}\text { User } \\
\text { friendly }\end{array}$ & Functionality \\
\hline LDpred $^{16}$ & $\begin{array}{l}\text { Bayesian } \\
\text { Shrinkage Prior }\end{array}$ & Python & Difficult & $\begin{array}{l}\text { Uses a prior on effect sizes and LD } \\
\text { information from an external reference } \\
\text { panel }\end{array}$ \\
\hline PRS-CS ${ }^{24}$ & $\begin{array}{l}\text { Bayesian } \\
\text { regression } \\
\text { framework }\end{array}$ & Python & Difficult & $\begin{array}{l}\text { Utilizes a high- dimensional Bayesian } \\
\text { regression framework, by placing a } \\
\text { continuous shrinkage (CS) prior on SNP } \\
\text { effect sizes }\end{array}$ \\
\hline EB-PRS ${ }^{19}$ & $\begin{array}{l}\text { Empirical Bayes } \\
\text { approach }\end{array}$ & $\mathrm{R}$ & Difficult & $\begin{array}{l}\text { A novel method that leverages information } \\
\text { for effect sizes across all the markers }\end{array}$ \\
\hline AnnoPred $^{20}$ & $\begin{array}{l}\text { Bayesian } \\
\text { Shrinkage Prior }\end{array}$ & Python & Difficult & $\begin{array}{l}\text { A framework that leverages diverse types of } \\
\text { genomic and epigenomic functional } \\
\text { annotations }\end{array}$ \\
\hline PRSice $^{36}$ & $\begin{array}{l}\text { Clumping }+ \\
\text { thresholding (C } \\
+\mathrm{T})\end{array}$ & $\mathrm{R}$ & Difficult & $\begin{array}{l}\text { For calculating, applying, evaluating and } \\
\text { plotting the results of PRS analysis }\end{array}$ \\
\hline PRSice $2^{37}$ & $\begin{array}{l}\text { Clumping } \\
\text { +thresholding } \\
(\mathrm{C}+\mathrm{T})\end{array}$ & $\mathrm{C}++, \mathrm{R}$ & Easy & $\begin{array}{l}\text { An efficient and scalable software program } \\
\text { for automating and simplifying PRS analyses } \\
\text { on large-scale data }\end{array}$ \\
\hline LDpred $^{38}$ & $\begin{array}{l}\text { Bayesian } \\
\text { Shrinkage }\end{array}$ & $\mathrm{R}$ & Difficult & $\begin{array}{l}\text { A faster and more robust implementation of } \\
\text { LDpred in } \mathrm{R} \text { package bigsnpr }\end{array}$ \\
\hline BSLMM $^{39}$ & $\begin{array}{l}\text { Bayesian } \\
\text { sparse linear } \\
\text { mixed model }\end{array}$ & $\mathrm{R}$ & Difficult & $\begin{array}{l}\text { Prior specification for the hyper-parameters } \\
\text { and a novel Markov chain Monte Carlo } \\
\text { algorithm for posterior inference }\end{array}$ \\
\hline BayesR $^{23}$ & $\begin{array}{l}\text { Hierarchical } \\
\text { Bayesian } \\
\text { Mixture Model }\end{array}$ & Fortran & Difficult & $\begin{array}{l}\text { Bayesian mixture model that } \\
\text { simultaneously allows variant discovery, } \\
\text { estimation of genetic variance explained by } \\
\text { all variants. }\end{array}$ \\
\hline $\begin{array}{l}\text { DPR } \\
\text { software }^{40}\end{array}$ & $\begin{array}{l}\text { Latent Dirichlet } \\
\text { process } \\
\text { regression } \\
\text { model }\end{array}$ & $\mathrm{C}++$ & Easy & $\begin{array}{l}\text { Dirichlet process regression to flexibly and } \\
\text { adaptively model the effect size distribution. }\end{array}$ \\
\hline SMTpred $^{41}$ & & Python & Difficult & $\begin{array}{l}\text { Combines SNP effects or individual scores } \\
\text { from multiple traits according to their } \\
\text { sample size, SNP-heritability }\left(h^{2}\right) \text { and genetic } \\
\text { correlation }\left(r_{G}\right) \text {. }\end{array}$ \\
\hline Lassosum $^{21}$ & $\begin{array}{l}\text { Penalised } \\
\text { Regression }\end{array}$ & $\mathrm{R}$ & Difficult & $\begin{array}{l}\text { A method for constructing PGS using } \\
\text { summary statistics and a reference panel in } \\
\text { a penalized regression framework. }\end{array}$ \\
\hline Plink $^{42}$ & $\begin{array}{l}p \text {-value } \\
\text { thresholding } \\
\text { approach }\end{array}$ & $\mathrm{C} / \mathrm{C}++$ & Easy & $\begin{array}{l}\text { Open-source } \mathrm{C} / \mathrm{C}++ \text { toolset for GWAS } \\
\text { analysis and research in population } \\
\text { genetics. }\end{array}$ \\
\hline
\end{tabular}

\section{Empirical Bayes PRS (EB-PRS) method}

The EB-PRS technique is an innovative method that relies on the Empirical Bayes theorem. It incorporates information across markers to strengthen prediction accuracy. ${ }^{19}$ By utilizing the predicted distribution of effect sizes, the EB-PRS technique tries to reduce prediction error. Suppose all the SNPs are independent, the optimum PRS value is given by:

$$
S=\beta^{T} X=\sum_{i=1}^{m} \beta_{i} X_{i}
$$

where $m$ denotes to the number of the all genotyped SNPs. The matrix $X_{i}$ stands for the genotypic value and $\beta_{i}$ is the logodds ratio $(\mathrm{OR})$ of the $i$ th variant. The equation below can be used to measure the log-OR: 
Table 2. Comparison of different approaches for performing PRS analyses.

\begin{tabular}{|c|c|c|c|c|}
\hline \multirow[t]{2}{*}{ Key factors } & \multicolumn{4}{|l|}{ Approaches } \\
\hline & $\begin{array}{l}p \text {-value } \\
\text { thresholding } \\
\text { with clumping }\end{array}$ & Penalised regression & $\begin{array}{l}\text { Clumping + } \\
\text { thresholding } \\
(\mathrm{C}+\mathrm{T})\end{array}$ & $\begin{array}{l}\text { Bayesian shrinkage } \\
\text { prior }\end{array}$ \\
\hline $\begin{array}{l}\text { Controlling for } \\
\text { Linkage } \\
\text { Disequilibrium }\end{array}$ & $\mathrm{N} / \mathrm{A}$ & $\begin{array}{l}\text { LD matrix is integral to } \\
\text { algorithm }\end{array}$ & Clumping & $\begin{array}{l}\text { Shrink effect sizes } \\
\text { with respect to LD }\end{array}$ \\
\hline $\begin{array}{l}\text { Shrinkage of GWAS } \\
\text { effect size } \\
\text { estimates }\end{array}$ & $P$-value threshold & $\begin{array}{l}\text { LASSO, Elastic Net, } \\
\text { penalty parameters } \\
\text { Bayesian }\end{array}$ & $\begin{array}{l}\text { P-value } \\
\text { threshold } \\
\text { standard }\end{array}$ & $\begin{array}{l}\text { Prior distribution, } \\
\text { e.g. fraction of causal } \\
\text { SNPs }\end{array}$ \\
\hline
\end{tabular}

where $f_{i 0}$ denotes the reference allele frequencies among the control samples and $f_{i 1}$ denotes the reference allele frequencies among the target. If $\beta_{i}=0$, that means the SNP is not correlated with the phenotype.

The actual values of effect sizes are generally unknown, thus they can be estimated empirically. Song et al. ${ }^{19}$ used the Empirical Bayes method to estimate $\beta$. The estimators can be equally derived from GWAS summary statistics. Unlike other improved genetic risk prediction methods which utilize effect size distributions for PRS computation, the EB-PRS does not require external panels. ${ }^{16,18,20,21}$ Also, the EB-PRS approach has theoretical superiority, resulting in a better PRS by lowering prediction error. The EB-PRS has recorded excellent performance in comparable to the other tool from following complex traits; Crohn's disease, celiac disease, Parkinson's disease, asthma, breast cancer, and type 2 diabetes. ${ }^{19}$ Furthermore, a significant improvement was recorded when tested against the unadjusted PRS method, P $+\mathrm{T}$, LDpred-inf, LDpred. ${ }^{18}$ Although The EB-PRS approach has demonstrated that it can generate superior results without adjusting any parameters or relying on external data, studies have shown that further improvement is possible with a reference panel. For instance, the LD information as used in LDpred. Also, to increase the prediction accuracy, Song et al. ${ }^{19}$ suggested that other available datasets such as GWAS summary statistics focused on functional annotations and genetically correlated traits could further improve EB-PRS accuracy.

Polygenic Risk Score-Continuous Shrinkage (PRS-CS) method

The PRS-CS is based on a Bayesian high-dimensional regression framework for polygenic modeling and prediction:

$$
Y_{N \times 1}=X_{N} \beta_{M \times 1}+\varepsilon_{N \times 1},
$$

where $N$ refers to the sample size and $M$ denotes the total number of the genetic markers. $Y$ represents a vector of phenotypes/traits and $X$ represents the genotype matrix. $\beta$ is a vector of effect sizes for the genetic markers and $\varepsilon$ is a vector of residuals. By assigning appropriate priors on the regression coefficients $\beta$ to impose regularization, the additive PRS value can be calculated using a posterior mean effect sizes. LDpred ${ }^{16}$ and the normal mixture model ${ }^{22,23}$ have $^{2}$ incorporated genome-wide markers with varying genetic architectures. The PRS-CS method aims to utilize a Bayesian regression framework and places a conceptually different class of priors (the continuous shrinkage (CS) priors) on SNP effect sizes. ${ }^{24}$ On the other hand, continuous shrinkage priors allow for marker-specific adaptive shrinkage. The amount of shrinkage applied to each genetic marker is adaptive to the strength of its associative signal in GWAS, which accommodates diverse underlying genetic architectures. Feng \& Smoller ${ }^{24}$ presented the PRS-CS-auto method, a fully Bayesian approach that enables automatic learning of a tuning parameter $\phi$, from GWAS summary statistics. Although analyses conducted from the Biobank indicate that for many disease phenotypes, the current GWAS sample sizes may not be large enough to accurately learn $\phi$ and the prediction accuracy of the PRS-CS-auto method may be lower than PRS-CS and LDpred. Nevertheless, simulation studies and quantitative trait analyses suggest that the PRS-CS-auto method can be useful when the size of the training dataset is large or when an independent validation set is difficult to acquire. Although the PRS-CS method provides a substantial improvement over the existing methods for polygenic prediction, ${ }^{16}$ the current prediction accuracy of the PRS value is still lower than what can be considered clinical utility. Much work is needed to advance the predictive performance and translational value of PRS methods. Recent studies argued that jointly modeling multiple genetically correlated traits and functional annotations in polygenic modeling are expected to increase the predictive performance of PRS methods. ${ }^{25-27}$ 
PRS methods based on shrinkage of GWAS effect size estimates

Since SNP effects are calculated with uncertainty and not all SNPs have an impact on the traits, unadjusted effect size estimates of all SNPs can lead to a low-estimated PRS with high standards error. ${ }^{17}$ Two shrinkage methods have been implemented to solve these problems; shrinkage of the effect estimates of all SNPs by adapted statistical techniques and use of $p$-value filtering thresholds as the criterion for inclusion of SNPs.

Shrinkage of the effect estimates of all SNPs by adapted statistical techniques: Some PRS methods performs shrinkage of all SNPs. These methods are typically apply shrinkage/regularisation techniques such as LASSO/ridge regression ${ }^{28}$ or Bayesian approaches performing shrinkages by prior distribution specification. ${ }^{16}$ Varying degrees of shrinkage may be accomplished under different methods or parameter settings. The most suitable shrinkages to be implemented depends on the underlying mixture of distributions of null and true effect size. PRS estimation is usually tailored over several (tuning) parameters since the optimum shrinkage parameters are a priori unknown. For example, it includes a setting for a fraction of causal variant ${ }^{16}$ in the case of LDpred.

p-value filtering thresholds as the criterion for inclusion of SNPs: In this process, the PRS includes significant SNPs with a P-value below a choosen threshold (e.g. $p$-value $<23$ e-05). This method shrinks all omitted SNPs to an estimated effect size of zero and does not perform shrinkage on the effect size estimates of the included SNPs. Since the optimum $p$ value threshold is a priori unknown, PRS is computed over a range of thresholds associated with each of the tested target traits and optimized appropriately for the prediction. This is similar to optimizing parameters in the systematic shrinkage approach and regarded as a parsimonious method of variable selection. It is efficient in performing the forward selection of variables (SNPs) using GWAS and p-value with the sizes depending on the $p$-value threshold increment. Therefore, this forward selection method is the chosen'optimal threshold'. Furthermore, PRS derived from another subset of the SNPs may be more predictive of the target trait. Considering the fact that GWAS focuses on millions of SNPs, the number of subsets of SNPs for the study could be too large.

\section{Linkage disequilibrium control}

Usually, association studies in GWAS are done individually. ${ }^{17}$ The power of GWAS can be enhanced by leveraging the results of several SNPs concurrently. ${ }^{29}$ Unfortunately, the raw data of all samples are not readily available. Researchers may need to take advantage of standard GWAS by considering either (i) SNPs are clumped such that the retained SNPs are almost independent of each other or (ii) all SNPs are included and the LD between them is adjusted. In the'standard' polygenic scoring approach, option $i$ is usually preferred and requires $p$-value thresholding. Option $i i$ is commonly used in methods that incorporate conventional methods of shrinkage ${ }^{21,16}$ (see Table 2). As for option $i$ without clumping, some researchers tend to apply the methods of $p$-value thresholding. Although breaking this presumption can lead to marginal losses in certain situations. ${ }^{21}$ Choi et al. ${ }^{17}$ suggested that clumping should be applied when GWAS estimates of nonshrunk effect sizes are available. The standard method tends to work when compared to more advanced approaches. ${ }^{21,16}$ It is possible that the clumping method captures conditionally independent effects. A critique of clumping for SNPs elimination in LD is that researchers usually use an arbitrarily selected correlation threshold. ${ }^{30}$ Thus, no technique is without arbitrary features. This could be an area for the potential development of the classical method.

\section{PRS approach based on clustering and decomposition of genetic variants}

PRS based variant decomposition focuses on decomposing or factorizing suitable genetic variants matrix into different components. This approach is mainly based on the use of an appropriate matrix decomposition technique. Contrary to traditional methods that compute PRS for a trait as the sum of effects from several genetic variants, this technique uses genetic risk for a single component to approximate risk for a weighted combination of relevant traits. Although there are many approaches to genetic variants decomposition, ${ }^{31-33}$ only truncated singular value decomposition (TSVD) and singular value decomposition (SVD) have been used in the context of PRS.

Aguirre et al. $^{34}$ and Chasman et al..$^{35}$ are the first to use genetic risk decomposition to derive polygenic scores. They both applied TSVD and SVD respectively to compute polygenic risk scores from genetic components. While it is similar to the traditional PRS in predictive ability, it also enables an appropriate assessment of drivers of genetic risk for the phenotype. For example, Aguirre et al. ${ }^{34}$ applied this method to body mass index and classified polygenic risk factors into overall health indicators, including sleep duration, alcohol, water intake, fat mass, fat-free mass. Consequently, they encouraged modeling PRS from the components of the decomposition of genetic risk association.

Let $W_{n \times m}$ be a sparse matrix of genetic associations with $n$ rows and $m$ columns, then TSVD can be performed on $W$ to identify different genetic components. The decomposition will lead to factors of three matrices which approximates $W$ : 
- A singular matrix for trait $U_{n \times c}$,

- A singular matrix for variant $V_{m \times c}$, and

- A diagonal matrix $S_{c \times c}$ of singular values. i.e., $W$.

Using the individual-level genotype vector $G_{m \times 1}$, component polygenic risk scores (cPRS) can be computed by applying matrices $U, S$, and $V$, using the following formula

$$
c P R S \approx S_{i} * V^{T} * G
$$

Finally, PRS can be defined by summing through the component PRS, using cPRS for each component, then;

$$
P R S=\sum_{i} U_{i j} * c P R S_{i}
$$

\section{PRS tools}

The next section will provide examples of some PRS tools that are commonly used to perform PRS analysis.

\section{Linkage Disequilibrium Pred (LDpred)}

This method estimates the posterior mean effect size of each marker of GWAS summary data using a priori effect sizes and LD information from an external reference panel. ${ }^{16}$ In this process, the inner products are re-weighted and the testsample genotypes are the posterior mean phenotype. The posterior mean phenotype is an optimum predictor under the model assumptions and a point-normal mixed distribution is used as the effect size prior, allowing for non-infinitesimal genetic structures. Heritability explained by the fraction of causative markers and genotypes are the two parameters of the prior. The heritability parameter is calculated using summary statistics from GWAS and takes into account sample noise and $\mathrm{LD}^{43}$

In an attempt to check the performance of LDpred in comparison to the method of pruning followed by thresholding, using five complex traits, including breast cancer, schizophrenia, muscular dystrophy, and coronary artery disease. GWAS summary statistics for large sample sizes ranging from 27,000 to 86,000 individuals and raw genotypes for an independent dataset validated, LDpred outperforms the other approach ${ }^{18}$ particularly at large sample sizes. For instance, the predicted $R^{2}$ rose from 20.1 percent to $25.3 \%$ and from $9.8 \%$ to $12.0 \%$ in a large dataset of schizophrenia and multiple sclerosis, respectively. Although the accuracy of the predictive values were lower in absolute terms in another study to predict schizophrenia risk in non-European validation populations of African and Asian heritage, similar observations were made for other approaches.

LDpred is a powerful tool that can be used for performing polygenic scores using summary statistics and LD information. ${ }^{44}$ However, one of its limitations is that its underlying algorithm assumes the existence of causal variants, which may result in limited predictive performance. In addition, its Gibbs sampler is sensitive to the model parameters for the large sample sizes. Moreover, LDpred can not predict PRS accurately for genomic regions with long-range LD, for instance, the human leukocyte antigen (HLA) region of Chromosome $6 .{ }^{23,25}$ However, long-range LD regions of the genome might contain many known disease-relevant variants. ${ }^{45,46}$ Privé et al. developed a new version of LDpred to address these shortcomings and improve its computational efficiency. ${ }^{38}$ This new version of LDpred has been implemented in the R package bigsnpr; see the next section.

\section{LDpred2}

LDpred2 is the improved version of LDpred tool by introducing new options to learn the effect accurately. For instance, the option sparse can estimate the effects that are 0 while the option auto can estimate the parameters from data and computes values for hyper-parameters $p$ and $h^{2}$. Due to these improvements, LDpred2 has been widely used to generate polygenic models with good predictive performance. ${ }^{47}$ However, LDpred 2 still has some issues regarding its stability. ${ }^{25,23}$ These issues contributed to the discrepancies in reported prediction accuracies. ${ }^{37,48}$ For instance, in contrast to LDpred, LDpred2 performs very well in the HLA regions but not for all traits as LDpred2 does not perform well for type 1 diabetes (T1D) and pure red cell aplasia (PRCA). LDpred2 performs poorly on T1D because T1D is mainly composed of large effects in the HLA region, while summary statistics typically have a small sample size. However, it is unknown 
why LDpred2 performs poorly, specifically for PRCA. Further studies are needed to understand why LDpred2 underperform in these two cases.

PRSice

PRSice, developed by Euesden et al. ${ }^{36}$ in 2015, was the first specialized PRS analysis program. PRSice is built in R and includes wrappers for bash data management scripts as well as PLINK-1.9 to speed up computation (Table 1). Using a list of $m$ SNPs and $n$ individuals from the 'target phenotypic' dataset, here, thegenotypes have some influence on the 'base phenotype'. If assessing the common genetic overlap of phenotype between samples/populations, the base and target phenotypes may be the same. A univariate regression on the base phenotype for each SNP, such as from genome-wide association research, can be used to estimate genotype effects (GWAS). For a SNP $i$, where $i=1,2, \ldots, m$, a $p$-value, $P_{i}$, is computed for the association between the SNP and genotypes, $G_{i}, j=\{0,1,2\}$ for individual $j$ where $j=1,2, \ldots, n$ and the phenotype. Under the standard additive assumption used in GWAS, a corresponding effect size for the effect of a unit increase in genotype Gij on the phenotype is estimated by $\beta_{i}$. The degree of estimate is used to determine which SNPs should be included in a PRS value. SNP $i$ will be included in in a PRS computation if $P i$ is less than a threshold, $P_{T}$, based on the $p$-value for their association with the base phenotype in a GWAS. Typically, PRS values are calculated at distinct $P_{T} p$-value thresholds.

At threshold $P_{T}$, the PRS value for individual $j$ can be calculated as:

$$
P R S_{P T, j}=\sum_{i=1}^{m} \beta_{i} G_{i, j} .
$$

The PRS value is computed across all individuals, yielding $n$ scores per $P_{T}$ threshold value. A suitable regression model could be used to assess the relationship between these PRS values and the target phenotype. The PRSice tool was created to fully automate PRS analyses, significantly enhancing PLINK-1.9's capabilities. ${ }^{49}$ Unless the genotypes have previously been imputed, there is generally some missing genotype data in real data. PLINK-1.9 fills in any missing data using mean allele frequencies. Nevertheless, it is not equipped to handle very large data sets. Hence a more memoryefficient approach is used in its advanced version, PRSice-2.

\section{PRSice-2}

PRSice-2 is an improved version of PRSice. It works with genotyped and imputed data, gives empirical association $p$ values that are free of overfitting inflation, supports numerous inheritance models, and analyzes numerous continuous and binary target traits at the same time. ${ }^{37}$ This technique simplifies the PRS analysis pipeline by eliminating intermediary files and doing all of the core computations in $\mathrm{C}++$, resulting in a significant decrease in execution time and memory use. Furthermore, while computing the PRS value, PRSice- 2 can immediately handle the BGEN imputed format and convert it to either best-guess genotypes or doses without producing a big intermediate file. While PRS values based on best-guess genotypes are produced using genotyped input, PRS values based on dose are derived using the following formula:

$$
P R S=\sum_{i}^{m} \beta_{i}\left(\sum_{j}^{2} \omega_{i j} X_{j}\right) .
$$

Where $\omega_{i j}$ is the probability of observing variant $j$, the value of $j \in\{0,1,2\}$, for the $i^{\text {th }} \mathrm{SNP} / \mathrm{variant}$; $m$ represents the number of SNPs/variants; and $\beta_{i}$ denotes the effect size of the $i^{\text {th }}$ variant estimated from the relevant base data set. A simulation study has been used to compare the performance of PRSice-2 to alternative polygenic score software lassosum $^{21}$ and LDpred $^{16}$ in terms of run time, memory usage and predictive power on servers equipped with 286 Intel 816824 core processors at $2.7 \mathrm{GHz}$ and $192 \mathrm{~GB}$ of RAM.

Based on a simulation study, PRSice-2 outperformed lassosum and LDpred in all circumstances. PRSice-2, in particular, can do full PRS analysis on 100,000 samples in 4 minutes, 179 times quicker than lassosum, which required 10 hours for the same task, and 241 times faster than LDpred, which took about 13 hours 27 minutes. Similarly, PRSice-2 uses substantially less memory than lassosum and LDpred, requiring less than $500 \mathrm{MB}$ for 100,000 samples against $11.2 \mathrm{~GB}$ for lassosum and 45.2 GB for LDpred.

In another study to compare its predictive power for quantitative traits with a heritability of 0.2 and a base sample size of 50,000, and a target sample size of 10,000, PRSice-2 resulted in PRS values that are higher than LDpred but not as high as lassosum. The details about how it performs, inspection and analyses can be found (here). While the PRS values obtained 
by PRSice-2 do not fully optimize prediction accuracy, the straightforward technique and use of fewer SNPs allow for a clearer understanding of the results when compared to approaches that employ all SNPs. ${ }^{50}$

Lassosum

Lassosum is an alternative method that uses summary statistical data to estimate PRS and takes LD into account by using reference panels ${ }^{21}$ based on the commonly used LASSO and elastic net regression. ${ }^{51,52}$ Consider the linear regression given below:

$$
y=X \beta+\varepsilon .
$$

For which $X$ represents a data matrix of $n$-by- $p$, and $y$ denotes a vector of the observed outcome. LASSO is a commonly used method for deriving $\beta$ estimates and y predictors, especially in cases where $\mathrm{p}$ is high and where it is rational to conclude that many $\beta$ are 0 . By minimizing the objective function, LASSO also obtains estimates of $\beta$ given $y$ and $X$. To test the efficiency of lassosum relative to LDpred, simulation studies were carried out using summary statistics accounting LD and Phase 1 data from Welcome Trust Case Control Consortium (WTCCC) for seven diseases. ${ }^{44}$ The outcome of LDpred, lassosum and simple soft-thresholding (setting $s=1$ in lassosum) was compared with most of the diseases in the WTCCC dataset, except for T1D where lassosum seem to outperform LDPred. The performance of LDpred and lassosum was comparable when the number of causal SNPs was 1,000 and the sample size was 11,200 for the simulated phenotypes, and both were superior to soft thresholding. Unlike lassosum, LDpred's performance was considerably reduced when the sample size was halved. The lassosum was not influenced in the same way when reducing the sample size by half. All methods performed equally when the number of causal SNPs was 25,000 and the sample size was 11,200 . The fact that summary statistics can be confounded by population stratification and population heterogeneity makes the real-life application of PRS difficult. These problems in the lassosum design were not considered. One possible issue with the use of meta-analytical summary statistics is that the original data produced by the summary statistics was an amalgamation of datasets around the world with corrections for population stratification. There is possibly no homogenous dataset suitable as a reference panel. Further research is required to explain the best approach.

Schork et al. ${ }^{53}$ have demonstrated that different genome regions have different false discovery rates, thus have different chances of being causally correlated with a phenotype. Genome annotation information can be used theoretically to enhance the performance. Similarly, it is possible to utilize the fact that certain phenotypes have common genetic determinants (pleiotropy) to improve PRS.

\section{PLINK SOFTWARE (Second-generation PLINK)}

PLINK 1 is an open-source $\mathrm{C} / \mathrm{C}++$ toolbox for population genetics research and GWAS data analysis. The increasing rise of data from imputation and whole-genome sequencing research necessitated the urgent need for speedier and scalable implementations of its essential functionalities. Furthermore, genotype likelihoods, phase information, and multiallelic variations are commonly found in GWAS and population-genetic data. However, these features cannot be handled by PLINK 1 primary data format cannot accommodate any of these. For these reasons, Chang et al. ${ }^{42}$ developed a new version called PLINK 1.9. This version features heavy use of bit-level parallelism, O (pn)-time/constant-space HardyWeinberg equilibrium computation, Fisher's exact testing, and a slew of other algorithmic enhancements. PLINK 1.9 speeds up most processes by 1-4 order of magnitude, allowing it to handle data sets that are too huge to store in RAM. The basic functional domains of PLINK 1.9 are identical to those of its predecessor, and it may be used as a drop-in replacement for existing scripts in most circumstances. Features, including the import/export of VCF, Oxford-format files, and fast cross-platform genomic relationship matrix calculators, have been included to facilitate easier interoperability with newer applications. Despite its computational advantages, PLINK 1.9 may still be an unsuitable tool for working with imputed genomic data due to the limitations of the PLINK 1 binary file format. To address this problem, the authors have developed PLINK 2.0, which features a new core file format capable of holding the bulk of the data generated by modern imputation systems.

\section{PRS tools in diverse populations}

Applying PRS analysis for multi-ethnic groups is still limited. Novel PRS methods have been developed to address the applicability of PRS analysis across ethnic groups.

Multi-ethnic PRS analysis: Multi-ethnic PRS analysis is a new PRS approach that combines PRS analysis based on two distinct populations. ${ }^{54}$ For instance, multi-ethnic PRS analysis could merge PRS analysis based on European training data with PRS analysis based on training data from another population. The multi-ethnic PRS approach computes PRS value given a target individual with genotypes $\mathrm{g}$ as follows: 


$$
P R S=\sum_{i=1}^{M} \widehat{b}_{i} g_{i}
$$

where $M$ denotes the number of individual's genetic markers, and the term $\widehat{b}_{i}$ is an estimate of effect sizes. For a multiethnic PRS analysis, this approach uses a linear combination of the two distinct PRS values and applying mixing weights parameters $\alpha_{i}$.

Linear unbiased predictors (BLUP): PRS analysis can be molded using the well-known approach of best linear unbiased predictors (BLUP). ${ }^{55}$ BLUP is used to consider and linearly model both random effects and fixed effects. It is also known as genomic best linear unbiased prediction (gBLUP). ${ }^{56}$ BLUP/gBLUP estimates PRS values using the following formula

$$
P R S=X \beta+g+\varepsilon,
$$

Where $\beta$ represents a vector of the fixed effects, $g$ denotes the total genetic effects in the base/training dataset, and $\varepsilon$ are the normally distributed residuals. To evaluate the fixed effects, BLUP considers an individual GWAS indicator, the top 5 principal components (PCs) derived with all samples together and/or a list of the significant SNPs. The BLUP approach is a computationally efficient algorithm. Nevertheless, the limitation of BLUP arose due to its requirement of the Individual-level genotype data. BLUP has been implemented in GCTA software (Genome-wide Complex Trait Analysis) . Moreover, it has been extended to XP-BLUP to model PRS values for admixed populations. ${ }^{56}$ Also, BLUP has been extended to MultiBLUP to include multiple random effects. ${ }^{57}$

Genetic Risk Scores Inference (GeRSI): GeRSI uses mixed models by combining fixed-effects models and randomeffects models for controlling population structure. ${ }^{58}$ GeRSI performs Gibbs sampling to estimate individuals' genetic risk score given the case-control study's genotypes under a random-effects model. GeRSI proposed conditional distributions of the genetic and environmental effect using the standard liability-threshold model. One limitation of GeRSI is that it requires individual-level genotypes which are not available to many bioinformaticians.

Cross-population BLUP (XP-BLUP): XP-BLUP is an extension of the BLUP method that can be applied to transethnic populations. ${ }^{58}$ XP-BLUP utilizes trans-ethnic information to improve PRS value predictive accuracy in minority populations. It combines the linear mixed-effects model (LMM) of the GeRSI method with the BLUP method.

\section{PRS analysis and population structure}

The main cause of false-positive genotype-phenotype associations in PRS analysis is from population genetic structure. ${ }^{59,17}$ In African populations with population structure, GWAS analysis techniques provide a significant rate of falsepositive results. ${ }^{60}$ These findings are influenced by the cohort's relatedness rather than variations that have an effect on the trait or disease risk. ${ }^{60}$ In general, structures in mating patterns induce structures in genetic variation closely associated with geographic location. Furthermore, risk factors due to the environmental exposure may be creating the possibility for correlations between genetic variations. Sul et al. ${ }^{60}$ have noted some confounding issues that are unique to GWAS research, such as 1) genetic artifacts such as errors on SNP array chips; 2) phenotypic and environmental diversity in the participants, such as gender, ancestry, and age; and 3) strategic ignorance about disease risk. ${ }^{59}$ These confounding factors affect the genomic composition of populations and are difficult to calculate as they are not openly evident. ${ }^{17,59,60}$ The characteristics examined are confounded by example and location. ${ }^{61,62}$ Usually, this issue is resolved in GWAS by modifying the $\mathrm{PCs}^{61}$ or by using mixed models. ${ }^{63}$

The population composition in the PRS study presents a possible great issue since there are a significant number of null variants in PRS estimation. For example, allele frequencies are systematically different between the base and target data. These can be obtained from genetic drift or genotyped variants. ${ }^{64}$ In addition, there is a danger that variations in null SNPs may result in the correlation between the PRS and target traits if the distributions of the environmental risk factors for the phenotype vary in base and target data or highly probable in most PRS studies. Even if the GWAS had completely regulated its population structure, confounding is possibly reintroduced. Correlated variations between the base and target data in allele frequencies and risk factors are not taken into consideration.

The regulation of structure in the PRS study should be adequate to prevent false-positives, if the base and target samples are drawn from the same or genetically similar populations. Choi et al. ${ }^{17}$ advised that there are drastic variations between 
populations in the distribution of PRS. ${ }^{64-66}$ Such observations do not indicate many differences between populations in etiology. Genuine differences are likely to contribute to geographical, cultural and selection pressure variations. It challenges the use of base and target data from different populations in PRS studies that do not tackle problems of possible uncertainty generated by geographical stratification. ${ }^{65}$ Therefore, by exploiting large sampling sizes, the effect can be obtained using subtle confounding. The issues of population structures are as important as the variations between individuals in the base and target populations in genetics and the environment. In the coming years, the discussion of generalizability of PRS methods across populations can be an active field. ${ }^{66,54}$

\section{Population bias in available genotyping platforms}

The PRS method that could be applied to diverse populations is still a challenging task. ${ }^{65}$ Many factors limit the application of PRS across diverse populations. These factors include:

- The limitation in the current genomics technologies

- LD distribution across diverse population

- The minor allele frequencies (MAF) distribution

- The distribution of the causal variants across diverse populations.

Current sequencing technologies are based on the European reference genome. Hence, the current genomics technologies are still not robust enough to capture genetic diversity among trans-ethnic populations. Studying LD patterns across diverse populations showed that the distribution of LD patterns plays a critical role in the underlying PRS value. ${ }^{67,68}$ Incorporating the information of LD patterns across diverse populations would increase PRS utilities among trans-ethnic populations. Moreover, the utility of PRS across diverse populations has limited the MAF across diverse populations. ${ }^{65,67}$ The differences in MAF variants across diverse populations will result in different variant selection, ${ }^{69}$ which will reflect PRS in calculations. Furthermore, to improve the utility of PRS across diverse populations, researchers should investigate the causal variants shared across multi-ethnic groups. ${ }^{70}$ Type 2 diabetes and body mass index account for $70-80 \%$ of African ancestry. However, because of variations in LD and allele frequency, the accuracy of African-based PRS was lower than that of European-based PRS. Some studies showed that Europeans' causal variants are also likely to be shared in African ancestry. ${ }^{71-73}$ Despite this, we can not generalize that the causal variants shared among trans-ethnic groups due to the limitation of representation of non-European populations, including sub-Saharan African communities. Previous approaches introduced to increase PRS accuracy in African populations prioritize the use of population-specific weighting and European discovered variants. However, due to the small sample sizes in African population, only moderate gains in accuracy are attainable. The example of a method that allows ethnic-specific weights to be included in their model is a two-component linear mixed model. In another study, Márquez-Luna et al. ${ }^{54}$ used Latino training data with limited sample size and publicly available large sample size European summary statistics to predict type 2 diabetes in a Latino cohort. When compared to previous methodologies, they achieved a relative improvement in prediction accuracy of more than $70 \%$. This technique was also used to predict height using European and African training data in an African UK Bio bank.

\section{Limitations of current PRS algorithms}

The methods for performing PRS vary based on two primary factors: (i) the list of SNPs to be used, and (ii) the weights to be used. Given the LD structure between SNPs, depending on the the trait's genetic architecture and GWAS discovery sample size, the appropriate technique for determining what weights to apply and which SNPs to choose will differ between traits. The following tools LDpred, LDpred funct, SBLUP, P+T, LDpred-Inf PRS-CS, SBayesR, and PRS-CSauto were employed in a comparative study to assess the PRS approaches in terms of their predictive potential. ${ }^{74}$ To accomplish this task, data from the major depressive disorder and Psychiatric Genomics Consortium working groups on schizophrenia were used. The results demonstrate that SBayesR outperforms the other tools in terms of speed and predicted accuracy. SBayesR, on the other hand, cannot produce converged solutions if the GWAS summary statistics have non-ideal features. While the benchmark $\mathrm{P}+\mathrm{T}$ approach performed the least, the other tools achieved nearly the same level of accuracy. In addition to being the best approach in this study, SBayesR has been designed to learn the genomic architecture from the GWAS attributes. Some of these approaches, including LDpred, use tuning cohorts to specify parameters for the target cohort. When the length of the Markov chain Monte Carlo chain increases for example in LDpred, the prediction accuracy improves. One drawback of such strategy is that the user will have to tune the model parameters. Substantial effort is currently ongoing to expand GWAS sample collection across demographic groups. Most of the existing tools use only samples of European ancestry in the comparative PRS study. As a result, further study is 
needed to assess the accuracy of alternative techniques in other ancestries and across ancestries, taking into account probable differences in genomic architectures and LD.

\section{The predictive power of PRS analysis}

Most articles within the current literature consider sample size as a milestone to power the PRS analysis. In 2013, Dudbridge estimated the predictive power of the polygenic score using results from several published studies. ${ }^{12}$ Dudbridge concluded that all published studies with a significant association of PRS values are statistically wellpowered. In addition, Dudbridge pointed out that the accuracy of the PRS analysis depends only on the size of the initial data set (training sample). Furthermore, he provided a mathematical model to estimate the statistical power of PRS value as a function of sample size. In 2014, Middeldorp et al. ${ }^{28}$ suggested that PRS analysis on a sample size of 2000 individuals is good enough to obtain a statistically powered PRS value. However, Dima and Breen in $2015^{75}$ demonstrated that a sample size of 1500 is enough to increase the predictive power to a statistically significant point. They stated that the predictive power of polygenic risk scores is not good enough for clinical utilities but it could be used as a biomarker for traits of interest within individuals. Recently, in 2017, Krapohl et al. ${ }^{5}$ introduced a multi-polygenic score that is capable of increasing the predictive power of PRS analysis. Regarding the relative accuracy of PRS values across ancestries, Yengo et al. ${ }^{76}$ proposed a theoretical model to estimate them. Their method utilized the frequencies of the minor alleles (MAF) in the two populations, the LD between the causal SNPs and the heritabilities. The authors assumed that causal variants are shared across ancestries however, their effect sizes might vary. Based on their model, Yengo et al. ${ }^{76}$ concluded that LD and MAF differences across ancestries explained 70-80\% of the loss of relative accuracy of European-based PRS value in African ancestry.

\section{PRS clinical utility}

PRS analysis has been successfully applied to estimate and identify individuals with genetic risk for many biological traits such as type 2 diabetes, breast cancer, and prostate cancer (See the extended data ${ }^{109}$ ). Most of these studies provide significant evidence of the success of PRS analysis in identifying patients who are at high risk of developing disease complications. Additionally, the primary strength of PRS analysis is its capability of stratifying individuals based on their probability of developing a disease. The biological power of PRS analysis arose due to its capacity to identify therapeutic and genomic pathways for type 2 diabetes, breast cancer, and prostate cancer. Moreover, applying PRS analysis on these traits showed that the reproducibility of PRS results is in the European population.

Nonetheless, one weakness of applying PRS analysis on these traits is its limited ability in detecting false-positive results. It is observed that most PRS studies are only available for European ancestries. Therefore, we can not apply them to non-European communities. In addition, performing PRS analysis on sizeable multi-ethnic data is indispensable for obtaining more accurate PRS values across populations. Furthermore, the possibility of applying PRS outcomes for personalized medicine requires robust validation procedures before broad clinical applications for multi-ethnic communities.

Understanding complex diseases and their clinical manifestations can be advanced significantly using accurate models for estimating PRS. The current PRS models can be used to forecast outcomes accurately. Disease subtypes and mechanisms that underpin within-trait diversity are not accounted for in PRS models, which might be important for analysis or therapeutic response. ${ }^{34,35,77,78}$ PRS models are used mainly to estimate clinical risk prediction for certain diseases, that can be extended to lifetime risk trajectories. ${ }^{79,80}$ Furthermore, PRS models can be implemented by clinical care authorities to decrease potential adverse health outcomes. Public health authorities can benefit from PRS models to control outbreaks of a particular disease by providing more efforts in high risk areas. PRS models can be used to define policies for administering the vaccination process. To use PRS accurately in clinical utilities as a personalized medicine tool, factors such as family history, rare monogenic mutations, ethnicity and ancestry, indirect genetic effects and gene-environment correlation should be considered. Refer to Table 3 for some commercial PRS kits that can be used for clinical utilities.

\section{PRS analysis on African populations}

The approach of the PRS analysis is yet to be extensively applied to study traits in African populations. For instance, searching PubMed for PRS in African populations on August 21, 2021 (see Figure 1 and Box 1), only gave 8,843 hits. This number represents about $4.553483 \%$ of total hits that were obtained without using the term' for African populations'. The traits studied using PRS analysis in African populations include types 1 \& 2 diabetes mellitus, depression, ischemic stroke, schizophrenia, sarcoidosis, Alzheimer's disease, obesity, insomnia disorder, post-traumatic stress and cancer. Undermentioned are some selected PRS studies in sub-Saharan African populations.

Ekoru and his colleagues investigated the genetic risk scores for cardiometabolic traits in several African ancestries, including sub-Saharan African populations. ${ }^{81}$ They concluded that the predictive power of the risk score is limited in African ancestry populations. They stated that this limitation is due to the insufficient diversity among their samples of 
Table 3. Examples of PRS kits for clinical utilities.

\begin{tabular}{|c|c|c|c|c|}
\hline Company & PRS Kit & Disease/Usage & Variants/Genes & Link \\
\hline \multirow[t]{7}{*}{ Illumina } & $\begin{array}{l}\text { Infinium } \\
\text { Global } \\
\text { Screening } \\
\text { Array v3.0 }\end{array}$ & $\begin{array}{l}\text { Autoimmune disorders, } \\
\text { childhood diseases, drug } \\
\text { responses. }\end{array}$ & 654,027 & \multirow[t]{7}{*}{$\begin{array}{l}\text { https://www. } \\
\text { illumina.com/ }\end{array}$} \\
\hline & $\begin{array}{l}\text { Infinium } \\
\text { Global } \\
\text { Screening } \\
\text { Array with } \\
\text { Multi-disease } \\
\text { drop }\end{array}$ & $\begin{array}{l}\text { Span of diseases: psychiatric, } \\
\text { neurological, cancer, } \\
\text { cardiometabolic, } \\
\text { autoimmune, } \\
\text { anthropometric. }\end{array}$ & $\approx 50 \mathrm{~K}$ variants & \\
\hline & Neuro Array & $\begin{array}{l}\text { Extensive neurodegenerative } \\
\text { disease. }\end{array}$ & $180 \mathrm{~K}$ & \\
\hline & Oncoarray & $\begin{array}{l}\text { Disease markers for a wide } \\
\text { range of tumor types. }\end{array}$ & 499,170 & \\
\hline & $\begin{array}{l}\text { DrugDev } \\
\text { Consortium } \\
\text { Array }\end{array}$ & Drugable targets. & 485,000 & \\
\hline & $\begin{array}{l}\text { H3Africa } \\
\text { Consortium } \\
\text { Array }\end{array}$ & $\begin{array}{l}\text { Epidemiological research: } \\
\text { Somatic mutations in cancer, } \\
\text { Disease defense, transplant } \\
\text { rejection, and autoimmune } \\
\text { disorder, drug responses. }\end{array}$ & 10,000 & \\
\hline & PsychArray & $\begin{array}{l}\text { Common psychiatric } \\
\text { disorders such as } \\
\text { schizophrenia, attention } \\
\text { deficit hyperactivity disorder, } \\
\text { bipolar disorder, major } \\
\text { depressive disorder, autism- } \\
\text { spectrum disorders, } \\
\text { obsessive-compulsive } \\
\text { disorder, anorexia nervosa } \\
\text { and Tourette's syndrome. }\end{array}$ & $\approx 30 \mathrm{~K}$ & \\
\hline 23andMe & $\begin{array}{l}\text { 1- Health + } \\
\text { Ancestry } \\
\text { Service } \\
\text { 2-23andMe + } \\
\text { Membership }\end{array}$ & $\begin{array}{l}\text { Several diseases, including } \\
\text { breast cancer, diabetes, } \\
\text { MUTYH-Associated Polyposis, } \\
\text { Late-Onset Alzheimer's } \\
\text { Disease, Parkinson's Disease, } \\
\text { lung and liver disease, } \\
\text { Chronic Kidney Disease, } \\
\text { Familial } \\
\text { Hypercholesterolemia, } \\
\text { anemia, nerve and heart } \\
\text { damage, and iron overload. }\end{array}$ & $\begin{array}{l}7,400-45,000 \text { markers } \\
\text { per chromosome }\end{array}$ & $\begin{array}{l}\text { https:// } \\
\text { www.23andme. } \\
\text { com/ }\end{array}$ \\
\hline Allelica & SCT-I & $\begin{array}{l}\text { Chronic diseases, including } \\
\text { coronary artery disease. }\end{array}$ & 1920136 & $\begin{array}{l}\text { https://www. } \\
\text { allelica.com/ }\end{array}$ \\
\hline $\begin{array}{l}\text { Ambry } \\
\text { Genetics }\end{array}$ & AmbryScore & Breast cancer. & 100 & $\begin{array}{l}\text { https://www. } \\
\text { ambrygen.com }\end{array}$ \\
\hline \multirow[t]{3}{*}{$\begin{array}{l}\text { Genetic } \\
\text { Technologies }\end{array}$} & $\begin{array}{l}\text { COVID-19 } \\
\text { Severity Risk } \\
\text { Test }\end{array}$ & COVID-19 & Not Provided & $\begin{array}{l}\text { https://www. } \\
\text { globenewswire. } \\
\text { com }\end{array}$ \\
\hline & $\begin{array}{l}\text { GeneType for } \\
\text { Breast Cancer }\end{array}$ & Breast Cancer. & $\begin{array}{l}77 \text { loci for Caucasian } \\
\text { women, } 74 \text { for African } \\
\text { American women and } \\
71 \text { for Hispanic women. }\end{array}$ & \\
\hline & $\begin{array}{l}\text { GeneType for } \\
\text { Colorectal } \\
\text { Cancer }\end{array}$ & Colorectal Cancer. & 45 & \\
\hline
\end{tabular}


Table 3. Continued

\begin{tabular}{|l|l|l|l|l|}
\hline Company & PRS Kit & Disease/Usage & Variants/Genes & Link \\
\hline Color & $\begin{array}{l}\text { Hereditary } \\
\text { Cancer Test }\end{array}$ & $\begin{array}{l}\text { Cancers: uterine, pancreatic, } \\
\text { ovarian, colon, melanoma, } \\
\text { breast, stomach, and } \\
\text { prostate cancers. }\end{array}$ & 30 genes & https://www. \\
color.com
\end{tabular}

genomic discovery. Therefore, they adjusted the ancestry-derived principal components to obtain up to 5-fold and 20-fold higher predictive power. They observed that the predictive power of genetic risk scores was higher among African Americans $(n=9139)$ and European Americans $(n=9594)$ relative to the sub-Saharan African populations $(n=5200)$. Based on this outcome, Ekoru and his colleagues concluded that PRS analysis performs poorly in sub-Saharan African populations. Furthermore, they recommended paying attention to the representation of the multi-ethnic population in genomic studies to improve the power of the genetic risk scores.

In 2020, Hayat and her colleagues investigated the genetic associations between serum low LDL-cholesterol levels and selected genetics variants in sub-Saharan African of four countries; Kenya, South Africa, Ghana and Burkina Faso. ${ }^{82}$ Using 1000 genomes data from the African populations, they selected four genes for their investigation ( $L D L R, A P O B$, PCSK9, and LDLRAP1). They performed genotyping of 19 SNPs using 1,000 participants in the Human Heredity and Health in Africa (H3Africa) AWI-Gen Collaborative Center (Africa, Wits-IN-DEPTH Partnership for GENomic studies). Although they used a limited number of variants, the outcome showed a significant association of these SNPs with lower LDL-cholesterol levels in sub-Saharan Africans.

In 2020, Cavazos and Witte proposed the inclusion of variants discovered from various populations to improve PRS transferability to diverse populations. ${ }^{83}$ They used both simulated data for the Yoruba group of the sub-Saharan African and European populations. They tested their findings on real data consisting of diabetes-free training samples of European ancestry $(n=123,665)$ and African descent $(n=7,564)$. They evaluated the performance of PRS analysis using genotype and phenotype data for a test (predictive) data set of European ancestry $(n=394,472)$ individuals of African origin from the UK Biobank $(n=5,886)$. Based on their findings, they concluded that incorporating variants selected from the European population will limit the accuracy of PRS values in non-Europeans populations including African communities. Also, they commented on the need for diverse GWAS data to improve PRS accuracy across populations.

In 2017, Marquez-Luna et al. ${ }^{54}$ proposed a multi-ethnic PRS analysis to improve risk prediction in diverse populations including African communities. To overcome the lack of enough training data for the African populations, the authors combined the training data from European samples and training data from the target population. We did not include their study because they did not state whether they used sub-Saharan African communities. This further highlights the challenge of performing PRS analysis in sub-Saharan African populations as a result of insufficient training data.

In 2017, Vassos et al. examined PRS values in a group of individuals with first-episode psychosis ${ }^{84}$ For the control data set, they combined African-European $(n=70)$ and a sample of sub-Saharan African ancestries $(n=828)$. Their finding showed that PRS value was more potent in Europeans, i.e. 9.4\% discriminative ability, than in Africans, i.e. only $1.1 \%$ discriminative ability in Africans.

PRS analysis is applied to investigate the risk score for prostate cancer. Prostate cancer is considered a complex genetic disease with high heritability which disproportionately affects men of African descent. ${ }^{85}$ A 1000 Genomes Project research that included seven African study sites and European males projected the risks of prostate cancer in urban African men. It was determined that the risks of prostate cancer are much more significant in African genomes than 
European genomes ( $p$-value $<2.2 \times 10 \mathrm{e}-16$, Wilcoxon rank-sum test). This continental level pattern is consistent with public health data. ${ }^{86}$ A further investigation was done by the team of MADCaP (Men of African Descent and Carcinoma of the Prostate Consortium) to study sites that portrayed a substantial amount of overlap in the PRS distributions of different African populations. Based on their findings, the investigators of MADCaP observed within-continent heterogeneity for the predicted risk of prostate cancer. Their findings showed that individuals from Dakar, Senegal have the lowest predicted risks of prostate cancer than other African study sites while individuals from Abuja, Nigeria have the highest predicted risks. The MADCaP team concluded that allele frequency differences at common disease-associated loci can contribute to population-level differences in prostate cancer risk.

\section{Challenges of PRS analysis for the African populations}

Many PRS methods have been developed and applied to test the risk score of individuals. Nevertheless, PRS analysis has not been used in the clinical field for the African population. There are still many limitations and challenges regarding the application of PRS analysis in the African population. One of these challenges is lack of sufficient data to perform PRS analysis. For instance, querying the term "sub-Saharan" in the GWAS Catalog repository, the search resulted in only 70 publications out of 4,628 papers. Considering that several publications might use the same GWAS data, we affirm that more GWAS experiments need to be done in sub-Saharan African populations. Lack of African population genetic data might be due to the following reasons: (i) African populations are not well presented in the reference genomes for variant calling and genotype calling; (ii) There is insufficient genetic diversity to capture the African specific variations in the average observable African population, i.e. sample sizes and the number of sub-population representations; (iii) there is lack of infrastructure and funding to perform GWAS experiments in many countries in Africa. Infectious diseases like malaria, tuberculosis, and HIV might still be prioritized by African scientists due to their public health importance and funding opportunities. Providing funding priority for infectious diseases is necessary for African communities as they account for a higher mortality rate in the continent.

Due to a lack of training and test data sets, some scientists choose to use training data from European samples that result in decreased PRS prediction accuracy. Therefore, PRS analysis is not widely applied for clinical utilities in Africa. The theory of genetics stated that when the genetic divergence in the target population and the original GWAS sample increases, the precision of the genetic risk prediction would decline. Several statistical discoveries are linked to this pattern: (i) The discovery of dominant genetic variations in the study population is favored by GWAS; (ii) even when the causative variants are the same, LD yields varied estimates of the marginal effect size for polygenic traits across populations; (iii) population-specific environmental and demographic differences. As a result, given the variety of the African population, the model developed elsewhere for PRS analysis does not fit for African sub-populations. Recent efforts to increase PRS accuracy in non-Europeans have prioritized the European discovered variants and populationspecific weighting. Due to a limitation of GWAS studies in African populations, this technique might be utilized to construct an African-specific PRS method that incorporates diverse sources of information. While the African-specific PRS approach aims to improve PRS accuracy, the shortage of long-term funds for GWAS research is another major obstacle in conducting and applying PRS research in the African context. Understudied populations, particularly in Africa provide possibility for genetic research. The common variants in these populations but uncommon or lacking in the European population could not be discovered using European sample sizes. SLC116A11 and HNF1A genes, for example are linked to type 2 diabetes, whereas APOL1 is linked to prostate cancer and end-stage kidney disease in AfricanAmericans. These issues are intractable with statistical techniques alone. Therefore, significant investment is required in African populations to yield similar-sized GWAS of biological traits.

As more data about genetic variation becomes available, the task of increasing the representation of African populations in the GWAS database has become increasingly essential. ${ }^{87,88}$ The inclusion of African multi-ethnic groups in GWAS analysis research is crucial for a more thorough, careful genetic variation and interpretation of the underpinnings of complex PRS analysis. ${ }^{87,88}$ In comparison to other under-represented populations, the average sample size of GWAS among Europeans continues to expand. PRS analysis in European populations has repeatedly failed to perform in African populations due to $\mathrm{LD}$, confounding of environmental factors across populations and differences in allelic architecture. $^{84,87-90,}$ The frequency of causative, risk allele, correlated variants, and disease prevalence all show substantialfrequency variation between populations. ${ }^{16,88}$ The magnitude and frequency of disease-causing genetic variants differ greatly among different populations including African ancestry. ${ }^{91}$ Overcoming these obstacles might lead to an effective clinical management, and specialized therapy for individuals and populations impacted by these complex disease and risk factors all of which would improve the health of those affected. ${ }^{87,91,92}$ Moreover, it could help in decreasing genotype imputation error, increase levels of tag-SNP portability, GWAS design, and effectively addressed GWAS analysis and interpretation in Africa populations. ${ }^{88,91}$

Therefore, African state authorities should be made aware of the challenges to make more funds available for genomic research. The funds should not be limited to the research institutes and principal investigators alone but they should 
equally provide scholarships (postgraduate programs like $\mathrm{PhD}$ ) and financial aids for young African researchers. We have some promising African research consortiums like The Pan-African Bioinformatics Network for the Human Heredity and Health in Africa (H3ABioNet, h3abionet.org) and the Human Heredity and Health in Africa (H3Africa, h3africa.org) that are contributing in this regard. However, their funds come from outside Africa. There are new regional Africa efforts like the World Bank-funded Africa Center of Excellence (ACE). It is important to state that these initiatives consist of few genomic research projects. A follow-up project to the H3Africa, dedicated to data science health research, entitled Harnessing Data Science for Health Discovery and Innovation in Africa (DS-I Africa) will soon commence.

Moreover, the lack of a pan-African genomic advisory board remains another challenge for genomic research in Africa. The existence of a research advisory board will help with transparency and establish ethical guidelines. These could open the window to get more grants from funding agencies such as the National Center for Biotechnology Information (NCBI). It is clear that without a rigorous ethical guide and transparency policies, it is hard to get long-term funds.

One more challenge of performing PRS for African populations is human migration. Environmental and social factors are the most critical drivers of disease risk than genetics in many cases so they must be effectively addressed. Benton et al. ${ }^{93}$ highlighted that early human migration out of Africa resulted in a higher genetic mutation rate, including diseaseassociated variants. Therefore, African populations do not carry the variants associated with disease at a higher frequency compared to non-African ancestries. As a result, given the genetic variation resulting from the diverse demographic history of the human populations, PRS prediction accuracy is still insufficient to generalize adequately across different populations, particularly for Africans. ${ }^{87,94}$ Furthermore, a lack of diversity in PRS development may contribute to existing health disparities among Africans. ${ }^{95,96}$ Therefore, consideration of environmental exposures and evolutionary histories must be key factors when performing PRS analysis.

\section{Application of PRS analysis on type 2 diabetes in African populations}

Diabetes mellitus prevalence was projected in 2019 to be 463 million globally, $4 \%$ of which are in African populations. ${ }^{97}$ In addition, Africa will witness the world's highest increase in diabetes prevalence by $2045 .{ }^{97,98}$ Currently, Africa has the most significant percentage of undiagnosed diabetics (59.7\%) in the world. As a result, immediate policies and resources for developing surveillance and an early detection approach to help Africa combat this pandemic has been initiated. ${ }^{99}$ The use of PRS for the early detection of people who are genetically predisposed to type 2 diabetes could significantly reduce the diabetes burden. According to data from European nations, individuals in the top $90 \%$ of the population had a 5.21fold higher likelihood of developing diabetes than those in the lowest $10 \%{ }^{100}$ Evidence has shown (coupled with a low GWAS study) that the transferability of polygenic scores developed in Europe decreases accuracy across diverse populations. ${ }^{87}$ Multi-ethnic PRS could be an alternative. However, the predictive performance of the African Americans and that of multi-ethnic PRS (who has about $80 \%$ African admixture) in continental Africans are yet to be examined. ${ }^{54,101}$ To ascertain this, Chikowore et al. aimed to see how well multi-ethnic, African-Americans, and European PRS would predict type 2 diabetes in Africans. ${ }^{99}$ For PRS development, the PRSice-2 software was used and the PRS with best result was chosen using area under the curve, i.e AUC and Nagelkerke R2. Finally, the results demonstrated that PRS derived from African Americans outperformed both multi-ethnic and European PRS in predicting type 2 diabetes. An earlier study of type 2 diabetes based on genetic risk score in Black South Africans used weight from Europeans $(\mathrm{OR}=1.21,95 \%$ $\mathrm{CI}){ }^{2}$ However, due to weights obtained from European-only studies, limited sample size, and use of only genotyped SNPs, this research was less predictive of Type 2 diabetes. Unlike previous work, this current study (Fatumo et al. ${ }^{99}$ ) took advantage of a larger sample size $(1,690)$, improved genome coverage and a multi-ethnic discovery dataset GWAS. All of these factors worked together to improve the PRS predictive ability. ${ }^{2}$

\section{PRS analysis on breast and prostate cancers in the continent of Africa}

Africa reportedly has the highest age-standardized death rate of breast cancer globally with sub-Saharan Africa having the highest prevalence rates. Although the occurrence in Africa was lower than in other continents, except for Asia, the mortality rate in Africa's sub-Saharan region (for example in Nigeria) was the highest in the world. ${ }^{102}$ Men of African origin have a greater prevalence and mortality rate from prostate cancer than men of other ethnic groups. Uganda has one of the highest prostate cancer incidence rates of all African nations. ${ }^{103}$ Genetic contributions to this difference are supported by evidence of genetic heterogeneity across populations. Breast and prostate cancer research in African populations can contribute to the elevated disease burden within this population by genetic risk factors. As a result, policymakers, academics and the general public must become aware of the rising threat that breast and prostate cancer can pose to Africa's growth. Early detection and stratification of women and men based on their risk of breast and prostate cancer using PRS could enhance screening and prevention strategies. Early detection of high disease risk individuals could also reduce the burden and threat to Africa's development. The application of PRS for breast and prostate cancer allows for early detection and risk stratification for recommendations and monitoring. ${ }^{104}$ To date, most of the GWAS SNPs were found almost entirely in European ancestry populations. They also demonstrate distinct patterns of relationship among the African populace. ${ }^{15,104}$ In addition, variants 
found in one community often do not apply to other populations of African ancestry. ${ }^{105}$ These contradictory findings may be attributed to various factors, including variations in allele frequencies and LD and differences in population characteristics within one ethnicity. As a result, there is a risk of PRS transferring PRS across populations. ${ }^{106}$ Some studies investigate PRS developed using GWAS data from various ancestry groups. ${ }^{107,108}$ For example, Belsky et al. ${ }^{107}$ constructed an obesity-based PRS relying on GWAS from European ancestry and discovered that it performed poorly in African Americans but worked well in European ancestry. ${ }^{107}$ On the other hand, Fritsche et al. ${ }^{105}$ concluded that, to some degree, cancer based PRS obtained from large Europeans ancestry GWAS may still be employed for disease risk stratification in populations if the limitations listed below are properly addressed:

- To accurately put an individual's PRS within their reference PRS distributions, a matched ancestry cohort with large control sample sizes is required.

- Non-European ancestry-derived PRS will be particularly useful for breast and prostate cancers because they have certain advantages over other traits: the high heritability is relatively high, normal in all ancestry groups, and publicity of summary statistics.

- Unlike individuals of diverse ancestries from different populations, the participants in the UK Biobank are mostly from the same country and healthcare accessibility and other risk factors are similar.

If summary statistics and large GWAS are available, Fritsche et al. ${ }^{103}$ argued that PRS development based on the same ancestral group might increase its predictive ability if summary statistics and large GWAS are available. Several methods are now being investigated to increase PRS predictive accuracy in African populations. If a large-scale GWAS for non-European populations are unavailable, these methods might be employed to improve PRS. On the other hand, these methods may incorporate the fact that SNP selection based on European based GWAS is applicable when employing European based GWAS effect sizes in ethnically mismatched populations. ${ }^{71,103}$

\section{Conclusion and future research}

There are several approaches under the umbrella of PRS analysis. GWAS are conducted on finite samples extracted from particular subsets of the human population. Moreover, the SNP effect size estimates are some combination of true effect and stochastic variation, thus producing'winner's curse' among the top-ranking associations and the estimated effects may not be well generalized to different populations. Furthermore, the correlation complicates the aggregation of SNP effects across the genome. Therefore, linkage disequilibrium holds the key to apply PRS analysis across ethnic groups. Thus, critical factors in the development of methods for calculating PRS values are

- The potential adjustment of GWAS estimated effect sizes e.g. via shrinkage and incorporation of their uncertainty.

- The tailoring of PRS values to target populations.

- The task of dealing with LD.

As members of the H3Africa consortium and the Associated Bioinformatics Consortium, H3ABioNet, (see h3abionet. org and https://sysbiolpgwas.waslitbre.org), we are working to extend existing methods to be applicable to African populations. Also, one future direction will be to develop an African-specific PRS method that combines the different sources of information. The information that we would consider to improve the current PRS methods include: (i) individual's ancestry information to include the diversity within Sub-Saharan populations; (ii) environmental risk factors to include the environmental diversity in Africa. Due to the variation in genetic architecture among trans-ethnic groups, we will consider incorporating information at the transcriptome level in the Sub-Saharan populations. Thus, providing a new PRS method that performs individual ancestry estimation and transcriptome risk score would improve the predictive value of the PRS besides providing insights into the molecular determinants of phenotypic traits, including rare diseases.

\section{Data availability}

Underlying data

No data is associated with this article. 
Extended data

Dryad: Polygenic Risk Score in Africa Populations: Progress and challenges, https://datadryad.org/stash/share/ btv5LlmVX0wVXdghkEOg1Thhua5VqQhugcG-825921Q. ${ }^{109}$

This project contains the following extended data:

- README file which provides information about the contents of the other file.

- A table contains selected studies in 2020 that demonstrate the PRS methods applied to diabetes type II, prostate cancer, and breast cancer.

Data are available under the terms of the Creative Commons Zero "No rights reserved" data waiver (CC0 1.0 Public domain dedication).

\section{Acknowledgements}

Authors acknowledge the logistical support of Mr. Babajide Ayodele. Covenant University provided the infrastructural support.

\section{References}

1. Bush WS: Genome-wide association studies.Encyclopedia of Bioinformatics and Computational Biology. Elsevier; 2019; pages 235-241.

Publisher Full Text

2. Gurdasani D, Carstensen T, Fatumo S, et al.: Uganda genome resource enables insights into population history and genomic discovery in africa. Cell. October 2019; 179(4): 984-1002.e36. PubMed Abstract | Publisher Full Text

3. Cantor RM, Lange K, Sinsheimer JS: Prioritizing GWAS results: A review of statistical methods and recommendations for their application. Am. J. Hum. Genet. 2010 January; 86(1): 6-22.

PubMed Abstract | Publisher Full Text

4. Zhang Q, Long Q, Ott J: ApriorigWAS, a new pattern mining strategy for detecting genetic variants associated with disease through interaction effects. PLoS Comput. Biol. June 2014; 10(6): e1003627.

PubMed Abstract | Publisher Full Text

5. Krapohl E, Patel $H$, Newhouse $S$, et al.: Multi-polygenic score approach to trait prediction. Mol. Psychiatry. August 2017; 23(5): 1368-1374.

PubMed Abstract | Publisher Full Text

6. Pasaniuc B, Price AL: Dissecting the genetics of complex traits using summary association statistics. Nat. Rev. Genet. November 2016; 18(2): 117-127.

Publisher Full Text

7. Chimusa ER, Dalvie S, Dandara C, et al.: Post genome-wide association analysis: dissecting computational pathway/ network-based approaches. Brief. Bioinform. April 2019; 20(2): network- $690-700$.

PubMed Abstract | Publisher Full Text

8. Buniello A, Macarthur JAL, Cerezo M, et al.: The NHGRI-EBI GWAS Catalog of published genome-wide association studies, targeted arrays and summary statistics 2019. Nucleic Acids Res. 2019; 47: D1005-D1012.

PubMed Abstract | Publisher Full Text

9. Beck T, Shorter T, Brookes AJ: GWAS Central: a comprehensive resource for the discovery and comparison of genotype and phenotype data from genome-wide association studies. Nucleic Acids Res. 10 2019; 48(D1): D933-D940. Publisher Full Text

10. Mailman MD, Feolo M, Jin Y, et al.: The NCBI dbGaP database of genotypes and phenotypes. Nat. Genet. October 2007; 39(10): 1181-1186. Publisher Full Text

11. Tryka KA, Hao L, Sturcke A, et al.: NCBI's database of genotypes and phenotypes: dbGaP. Nucleic Acids Res. December 2013; 42(D1):
D975-D979.

Publisher Full Text

12. Dudbridge F: Power and predictive accuracy of polygenic risk scores. PLoS Genet. March 2013; 9(3): e1003348.

PubMed Abstract | Publisher Full Text

13. Lewis $\mathrm{CM}$, Vassos E: Prospects for using risk scores in polygenic medicine. Genome Med. November 2017; 9(1): 96. PubMed Abstract | Publisher Full Text

14. Bramer WM, De Jonge GB, Rethlefsen ML, et al.: A systematic approach to searching: an efficient and complete method to develop literature searches. oct 2018; 106(4).

15. Chatterjee N, Wheeler B, Sampson J, et al.: Projecting the performance of risk prediction based on polygenic analyses of genome-wide association studies. Nat. Genet. March 2013; 45(4): 400-405.

Publisher Full Text | PubMed Abstract

16. Vilhjálmsson BJ, Yang J, Finucane HK, et al.: Modeling linkage disequilibrium increases accuracy of polygenic risk scores. $\mathrm{Am}$. J. Hum. Genet. October 2015; 97(4): 576-592. PubMed Abstract | Publisher Full Text

17. Choi SW, Mak TS-H, O'Reilly PF: Tutorial: a guide to performing polygenic risk score analyses. Nat. Protoc. July 2020; 15(9): 2759-2772.

PubMed Abstract | Publisher Full Text

18. So H-C, Sham PC: Improving polygenic risk prediction from summary statistics by an empirical bayes approach. Sci. Rep. February 2017; 7(1) PubMed Abstract | Publisher Full Text

19. Song $S$, Jiang $W$, Hou $L$, et al.: Leveraging effect size distributions to improve polygenic risk scores derived from summary statistics of genome-wide association studies. PLOS Comput. Biol. February 2020; 16(2): e1007565. PubMed Abstract | Publisher Full Text

20. Yiming $\mathrm{H}$, Qiongshi $\mathrm{L}$, Powles $\mathrm{R}$, et al.: Leveraging functional annotations in genetic risk prediction for human complex diseases. PLoS Comput. Biol. June 2017; 13(6): e1005589.

Publisher Full Text

21. Mak TSH, Porsch RM, Choi SW, et al.: Polygenic scores via penalized regression on summary statistics. Genet. Epidemiol. May 2017 41(6): 469-480. PubMed Abstract | Publisher Full Text

22. Zhang Y, Qi G, Park J-H, et al.: Estimation of complex effect-size distributions using summary-level statistics from genome-wide association studies across 32 complex traits. Nat. Genet. August 2018; 50(9): 1318-1326.

PubMed Abstract | Publisher Full Text 
23. Lloyd-Jones LR, Zeng J, Sidorenko J, et al.: Improved polygenic prediction by bayesian multiple regression on summary statistics. Nat. Commun. November 2019; 10(1): 5086. PubMed Abstract | Publisher Full Text

24. Ge T, Chen C-Y, Ni Y, et al.: Polygenic prediction via bayesian regression and continuous shrinkage priors. Nat. Commun. April 2019; 10(1): 1776

PubMed Abstract | Publisher Full Text

25. Márquez-Luna C, Gazal S, Loh P-R, et al.: LDpred-funct: incorporating functional priors improves polygenic prediction accuracy in UK biobank and 23andme data sets. July 2018.

26. Shi J, Park J-H, Duan J, et al.: Winners curse correction and variable thresholding improve performance of polygenic risk modeling based on genome-wide association study summary-level data. PLoS Genet. December 2016; 12(12): e1006493. PubMed Abstract | Publisher Full Text

27. Turley P, Walters RK, Maghzian O, et al.: Author correction: Multitrait analysis of genome-wide association summary statistics using MTAG. Nat. Genet. June 2019; 51(8): 1295-1295. PubMed Abstract | Publisher Full Text

28. Wray NR, Lee SH, Mehta D, et al.: Research review: Polygenic methods and their application to psychiatric traits. J. Child Psychol. Psychiatry. August 2014; 55(10): 1068-1087. PubMed Abstract | Publisher Full Text

29. Loh P-R, Kichaev G, Gazal S, et al.: Mixed-model association for biobank-scale datasets. Nat. Genet. June 2018; 50(7): 906-908. PubMed Abstract | Publisher Full Text

30. Wray NR, Yang J, Hayes BJ, et al.: Pitfalls of predicting complex traits from SNPs. Nat. Rev. Genet. June 2013; 14(7): 507-515. PubMed Abstract | Publisher Full Text

31. Tanigawa Y, Li J, Justesen JM, et al.: Components of genetic associations across 2,138 phenotypes in the UK biobank highlight adipocyte biology. Nat. Commun. sep 2019; 10(1): 4064 PubMed Abstract | Publisher Full Text

32. Zhao J, Feng QP, Patrick W, et al.: Using topic modeling via nonnegative matrix factorization to identify relationships between genetic variants and disease phenotypes: $A$ case study of lipoprotein(a) (LPA). PLoS One. feb 2019; 14(2): e0212112. PubMed Abstract | Publisher Full Text

33. Huseby CJ, Delvaux E, Coleman PD: A singular value decomposition algorithm to identify early dysfunctional molecular pathways in alzheimer's disease. Alzheimer's amp. Dementia. dec 2020; 16(S2)

Publisher Full Text

34. Aguirre $M$, Tanigawa $Y$, Venkataraman GR, et al.: Polygenic risk modeling with latent trait-related genetic components. Eur. J. Hum. Genet. feb 2021; 29: 1071-1081. PubMed Abstract | Publisher Full Text

35. Chasman DI, Giulianini F, Demler OV, et al.: Pleiotropy-based decomposition of genetic risk scores: Association and interaction analysis for type 2 diabetes and CAD. Am. J. Hum. Genet. may 2020; 106(5): 646-658. PubMed Abstract | Publisher Full Text

36. Euesden J, Lewis CM, O'Reilly PF: PRSice: Polygenic risk score software. Bioinformatics. December 2015; 31(9): 1466-1468. PubMed Abstract | Publisher Full Text

37. Choi SW, OReilly PF: PRSice-2: Polygenic risk score software for biobank-scale data. GigaScience. July 2019; 8(7) PubMed Abstract | Publisher Full Text

38. Privé F, Arbel J, Vilhjálmsson BJ: LDpred2: better, faster, stronger. April 2020.

39. Yang S, Zhou X: Accurate and scalable construction of polygenic scores in large biobank data sets. Am. J. Hum. Genet. May 2020; 106 (5): 679-693.

PubMed Abstract | Publisher Full Text

40. Zeng $P$, Zhou $X$ : Non-parametric genetic prediction of complex traits with latent dirichlet process regression models. Nat. Commun. September 2017; 8(1). Publisher Full Text

41. Maier RM, Zhu Z, Lee SH, et al.: Improving genetic prediction by leveraging genetic correlations among human diseases and traits. Nat. Commun. March 2018; 9(1): 989. PubMed Abstract | Publisher Full Text

42. Chang CC, Chow CC, Tellier LCAM, et al.: Second-generation PLINK: rising to the challenge of larger and richer datasets. Gigascience. February 2015; 4(1): 7

PubMed Abstract | Publisher Full Text

43. Finucane HK, Bulik-Sullivan B, Gusev A, et al.: Partitioning heritability by functional annotation using genome-wide association summary statistics. Nat. Genet.47(11): 1228-1235, September 2015.

PubMed Abstract | Publisher Full Text
44. Vilhjálmsson BJ, Yang J, Finucane HK, et al.: Modeling linkage disequilibrium increases accuracy of polygenic risk scores. $\mathrm{Am}$. J. Hum. Genet. October 2015; 97(4): 576-592.

PubMed Abstract | Publisher Full Text

45. Mokhtari R, Lachman HM: The major histocompatibility complex (MHC) in schizophrenia: A review. Journal of Clinical \& Cellular Immunology. 2016; 07(06).

PubMed Abstract | Publisher Full Text

46. Matzaraki V, Kumar V, Wijmenga C, et al.: The MHC locus and genetic susceptibility to autoimmune and infectious diseases. Genome Biol. April 2017; 18(1): 76.

PubMed Abstract | Publisher Full Text

47. Khera AV, Chaffin M, Aragam KG, et al.: Seung Hoan Choi, Pradeep Natarajan, Eric S. Lander, Steven A. Lubitz, Patrick T. Ellinor, and Sekar Kathiresan. Genome-wide polygenic scores for common diseases identify individuals with risk equivalent to monogenic mutations. Nat. Genet. August 2018; 50(9): 1219-1224. PubMed Abstract | Publisher Full Text

48. Ge T, Chen C-Y, Ni Y, et al.: Polygenic prediction via bayesian regression and continuous shrinkage priors. Nat. Commun. April 2019; 10(1): 1776.

PubMed Abstract | Publisher Full Text

49. Chang CC, Chow CC, Tellier LCAM, et al.: Second-generation PLINK: rising to the challenge of larger and richer datasets. GigaScience. oct 2014.

50. Cecile A, Janssens JW, Joyner MJ: Polygenic Risk Scores That Predict Common Diseases Using Millions of Single Nucleotide Polymorphisms: Is More, Better?. Clin. Chem. May 2019; 65(5): 609-611.

PubMed Abstract | Publisher Full Text

51. Tibshirani R: Regression shrinkage and selection via the lasso. Journal of the Royal Statistical Society. Series B (Methodological). 1996; 58(1): 267-288. Publisher Full Text

52. Zou H, Hastie $\mathrm{T}$ : Regularization and variable selection via the elastic net. Journal of the Royal Statistical Society: Series B (Statistical Methodology). April 2005; 67(2): 301-320. Publisher Full Text

53. Schork AJ, Thompson WK, Pham P, et al.: All SNPs are not created equal: Genome-wide association studies reveal a consistent pattern of enrichment among functionally annotated SNPS. PLoS Genet. April 2013; 9(4): e1003449. PubMed Abstract | Publisher Full Text

54. Márquez-Luna C, Loh P-R, Price AL: Multiethnic polygenic risk scores improve risk prediction in diverse populations. Genet. Epidemiol. November 2017; 41(8): 811-823. PubMed Abstract | Publisher Full Text

55. Chen C-Y, Han J, Hunter DJ, et al.: Explicit modeling of ancestry improves polygenic risk scores and BLUP prediction. Genet. Epidemiol. May 2015; 39(6): 427-438. PubMed Abstract| Publisher Full Text

56. Clark SA, van der Werf J: Genomic best linear unbiased prediction (gBLUP) for the estimation of genomic breeding values. Methods in Molecular Biology. Humana Press; 2013; pages 321-330. Publisher Full Text

57. Speed D, Balding DJ: MultiBLUP: improved SNP-based prediction for complex traits. Genome Res. June 2014; 24(9): 1550-1557. PubMed Abstract | Publisher Full Text

58. Golan D, Rosset $\mathrm{S}$ : Effective genetic-risk prediction using mixed models. Am. J. Hum. Genet. October 2014; 95(4): 383-393. PubMed Abstract | Publisher Full Text

59. Chen D, Liu C, Xie J: Multi-locus test and correction for confounding effects in genome-wide association studies. Int. J. Biostat. November 2016; 12(2) PubMed Abstract | Publisher Full Text

60. Sul HH, Martin LS, Eskin E: Population structure in genetic studies: Confounding factors and mixed models. PLOS Genet. December 2018; 14(12): e1007309. PubMed Abstract | Publisher Full Text

61. Price AL, Patterson NJ, Plenge RM, et al.: Principal components analysis corrects for stratification in genome-wide association studies. Nat. Genet. July 2006; 38(8): 904-909.

62. Astle W, Balding DJ: Population structure and cryptic relatedness in genetic association studies. Stat. Sci. November 2009; 24(4): 451-471. Publisher Full Text

63. Price AL, Zaitlen NA, Reich D, et al.: New approaches to population stratification in genome-wide association studies. Nat. Rev. Genet. June 2010; 11(7): 459-463. PubMed Abstract | Publisher Full Text

64. Kim MS, Patel KP, Teng AK, et al.: Genetic disease risks can be misestimated across global populations. Genome Biol. November 
2018; 19(1): 179

PubMed Abstract | Publisher Full Text

65. Martin AR, Gignoux CR, Walters RK, et al.: Human demographic history impacts genetic risk prediction across diverse populations. Am. J. Hum. Genet. April 2017; 100(4): 635-649. PubMed Abstract | Publisher Full Text

66. Duncan L, Shen $\mathrm{H}$, Gelaye B, et al.: Analysis of polygenic risk score usage and performance in diverse human populations. Nat. Commun. July 2019; 10(1): 3328

PubMed Abstract | Publisher Full Text

67. Shi H, Burch KS, Johnson R, et al.: Localizing components of shared transethnic genetic architecture of complex traits from GWAS summary data. Am. J. Hum. Genet. June 2020; 106(6): 805-817. PubMed Abstract | Publisher Full Text

68. Morgante F, Huang W, Maltecca C, et al.: Effect of genetic architecture on the prediction accuracy of quantitative traits in samples of unrelated individuals. Heredity. February 2018; 120(6): 500-514.

PubMed Abstract | Publisher Full Text

69. Lam M, Chen C-Y, Li Z, et al.: Comparative genetic architectures of schizophrenia in east asian and european populations. Nat. Genet. November 2019; 51(12): 1670-1678. PubMed Abstract | Publisher Full Text

70. Cavazos TB, Witte JS: Inclusion of variants discovered from diverse populations improves polygenic risk score transferability. Human Genetics and Genomics Advances. January 2021; 2(1): 100017.

PubMed Abstract | Publisher Full Text

71. Coram MA, Fang H, Candille SI, et al.: Leveraging multi-ethnic evidence for risk assessment of quantitative traits in minority populations. Am. J. Hum. Genet. August 2017; 101(2): 218-226. PubMed Abstract | Publisher Full Text

72. Marnetto D, Pärna K, Läll K, et al.: Ancestry deconvolution and partial polygenic score can improve susceptibility predictions in recently admixed individuals. Nat. Commun. April 2020; 11(1): 1628 .

PubMed Abstract | Publisher Full Text

73. Bitarello $B D$, Mathieson I: Polygenic scores for height in admixed populations. G3 (Bethesda). September 2020; 10(11): 4027-4036. PubMed Abstract | Publisher Full Text

74. Ni G, Zeng J, Revez JR, et al.: A comprehensive evaluation of polygenic score methods across cohorts in psychiatric disorders. September 2020.

75. Dima D, Breen $\mathrm{G}$ : Polygenic risk scores in imaging genetics: Usefulness and applications. J. Psychopharmacol. May 2015; 29(8): 867-871.

PubMed Abstract | Publisher Full Text

76. Wang Y, Guo J, Ni G, et al:: Theoretical and empirical quantification of the accuracy of polygenic scores in ancestry divergent populations. Nat. Commun. July 2020; 11(1) Publisher Full Text

77. Torkamani A, Wineinger NE, Topol EJ: The personal and clinical utility of polygenic risk scores. Nat. Rev. Genet. May 2018; 19(9): 581-590.

PubMed Abstract | Publisher Full Text

78. Roberts MC, Khoury MJ, Mensah GA: Perspective: The clinical use of polygenic risk scores: Race, ethnicity, and health disparities. Ethn. Dis. July 2019; 29(3): 513-516. PubMed Abstract | Publisher Full Text

79. Lambert SA, Abraham G, Inouye M: Towards clinical utility of polygenic risk scores. Hum. Mol. Genet. July 2019; 28(R2): R133-R142.

PubMed Abstract | Publisher Full Text

80. Jia G, Lu Y, Wen W, et al.: Evaluating the utility of polygenic risk scores in identifying high-risk individuals for eight common cancers. JNCI Cancer Spectrum. March 2020; 4(3) PubMed Abstract | Publisher Full Text

81. Ekoru K, Adeyemo AA, Chen G, et al.: Genetic risk scores for cardiometabolic traits in sub-saharan african populations. May 2020.

82. Hayat M, Kerr R, Bentley AR, et al.: Genetic associations between serum low LDL-cholesterol levels and variants in LDLR, APOB PCSK9 and LDLRAP1 in african populations. PLoS One. February 2020; 15(2): e0229098.

PubMed Abstract | Publisher Full Text

83. Cavazos TB, Witte JS: Inclusion of variants discovered from diverse populations improves polygenic risk score transferability. May 2020.

84. Vassos E, Di Forti M, Coleman J, et al.: An examination of polygenic score risk prediction in individuals with first-episode psychosis. Biol. Psychiatry. March 2017; 81(6): 470-477.

PubMed Abstract | Publisher Full Text
85. Rebbeck TR: Prostate cancer genetics: Variation by race ethnicity, and geography. Semin. Radiat. Oncol. January 2017; 27(1): 3-10. PubMed Abstract | Publisher Full Text

86. Bray F, Ferlay J, Soerjomataram I, et al.: Global cancer statistics 2018: GLOBOCAN estimates of incidence and mortality worldwide for $\mathbf{3 6}$ cancers in $\mathbf{1 8 5}$ countries. CA Cancer J. Clin. September 2018; 68(6): 394-424.

PubMed Abstract | Publisher Full Text

87. Martin AR, Kanai M, Kamatani Y, et al.: Clinical use of current polygenic risk scores may exacerbate health disparities. Nat. Genet. mar 2019; 51(4): 584-591.

PubMed Abstract | Publisher Full Text

88. Peprah E, Huichun X, Tekola-Ayele F, et al.: Genome-wide association studies in africans and african americans: Expanding the framework of the genomics of human traits and disease. Public Health Genomics. November 2014; 18(1): 40-51. PubMed Abstract | Publisher Full Text

89. Haga SB: Impact of limited population diversity of genome-wide association studies. Genet. Med. January 2009; 12(2): 81-84. Publisher Full Text

90. Maas $P$, Barrdahl $M$, Joshi $A D$, et al.: Breast cancer risk from modifiable and nonmodifiable risk factors among white women in the united states. JAMA Oncol. October 2016; 2(10): 1295-1302. PubMed Abstract | Publisher Full Text

91. Rosenberg NA, Huang L, Jewett EM, et al.: Genome-wide association studies in diverse populations. Nat. Rev. Genet. May 2010; 11(5): 356-366.

PubMed Abstract | Publisher Full Text

92. Li Z, Chen J, Yu H, et al.: Genome-wide association analysis identifies $\mathbf{3 0}$ new susceptibility loci for schizophrenia. Nat. Genet. October 2017; 49(11): 1576-1583. PubMed Abstract | Publisher Full Text

93. Benton ML, Abraham A, LaBella AL, et al.: The influence of evolutionary history on human health and disease. Nat. Rev. Genet. January 2021; 22(5): 269-283. PubMed Abstract | Publisher Full Text

94. Sirugo G, Williams SM, Tishkoff SA: The missing diversity in human genetic studies. Cell. March 2019; 177(1): 26-31. Publisher Full Text

95. Popejoy $A B$, Fullerton $\mathrm{SM}$ : Genomics is failing on diversity. Nature. October 2016; 538(7624): 161-164.

PubMed Abstract | Publisher Full Text | Free Full Text

96. Hindorff LA, Bonham VL, Brody LC, et al.: Prioritizing diversity in human genomics research. Nat. Rev. Genet. November 2017; 19(3): 175-185.

PubMed Abstract | Publisher Full Text

97. Saeedi P, Petersohn I, Salpea P, et al.: Global and regional diabetes prevalence estimates for $\mathbf{2 0 1 9}$ and projections for $\mathbf{2 0 3 0}$ and 2045: Results from the international diabetes federation diabetes atlas, 9th edition. Diabetes Res. Clin. Pract. nov 2019; 157: 107843.

Publisher Full Text

98. Ekoru K, Doumatey A, Bentley AR, et al.: Type 2 diabetes complications and comorbidity in sub-saharan africans. EClinicalMedicine. nov 2019; 16: 30-41. PubMed Abstract | Publisher Full Text

99. Chikowore T, Ekoru K, Vujkovic M, et al.: Polygenic prediction of type 2 diabetes in continental africa. Feb 2021.

100. Vujkovic M, Keaton JM, Lynch JA, et al.: Discovery of $\mathbf{3 1 8}$ new risk loci for type 2 diabetes and related vascular outcomes among 1.4 million participants in a multi-ancestry meta-analysis. Nat. Genet. Jun 2020; 52(7): 680-691. PubMed Abstract | Publisher Full Text

101. Zakharia F, Basu A, Absher D, et al.: Characterizing the admixed african ancestry of african americans. Genome Biol. 2009; 10(12): R141.

PubMed Abstract | Publisher Full Text

102. Torre LA, Bray F, Siegel RL, et al.: Global cancer statistics, 2012. CA Cancer J. Clin. Feb 2015; 65(2): 87-108. PubMed Abstract | Publisher Full Text

103. Fritsche LG, Ma Y, Zhang D, et al.: On cross-ancestry cancer polygenic risk scores. Mar 2021.

104. Zhang YD, Hurson AN, Zhang $\mathrm{H}$, et al.: Assessment of polygenic architecture and risk prediction based on common variants across fourteen cancers. Nat. Commun. Jul 2020; 11(1).

105. Fritsche LG, Patil S, Beesley LJ, et al.: Cancer PRSweb: An online repository with polygenic risk scores for major cancer traits and their evaluation in two independent biobanks. Am. J. Hum. Genet. Nov 2020; 107(5): 815-836. PubMed Abstract | Publisher Full Text 
106. Han $Y$, Hazelett $D J$, Wiklund $F$, et al.: Integration of multiethnic fine-mapping and genomic annotation to prioritize candidate functional SNPs at prostate cancer susceptibility regions. Hum.

Mol. Genet. Jul 2015; 24(19): 5603-5618.

Publisher Full Text

107. Belsky DW, Moffitt TE, Sugden K, et al.: Development and evaluation of a genetic risk score for obesity. Biodemography Soc. Biol. Jan 2013; 59(1): 85-100.

PubMed Abstract | Publisher Full Text
Grinde KE, Qi Q, Thornton TA, et al.: Generalizing polygenic risk scores from europeans to hispanics/latinos. Genet. Epidemiol. Oct 2018; 43(1): 50-62.

PubMed Abstract | Publisher Full Text

109. Adebiyi, et al.: Polygenic Risk Score in Africa Populations: Progress and challenges. Dryad [dataset]. 2021. Reference Source 


\section{Open Peer Review}

\section{Current Peer Review Status: ? ?}

\section{Version 1}

Reviewer Report 22 June 2022

https://doi.org/10.5256/f1000research.80186.r137670

(C) 2022 Lewis C. This is an open access peer review report distributed under the terms of the Creative Commons Attribution License, which permits unrestricted use, distribution, and reproduction in any medium, provided the original work is properly cited.

\section{Cathryn M. Lewis}

Social, Genetic and Developmental Psychiatry Centre \& Department of Medical and Molecular Genetics, King's College London, London, UK

\section{General:}

Adam et al. provide an extensive review of the important topic of PRS methods, and their applications in African ancestry populations. The paper summarises the various approaches to calculating PRS and provides a fair assessment of the advantages and disadvantages of each method. It also describes the challenges associated with calculating PRS in African populations and the approaches currently being undertaken to address these. The paper is comprehensive and a useful addition to the literature, pulling together a large amount of information across methods for calculating polygenic scores, and their applications in African populations.

\section{Major comments:}

To make the application of polygenic scores more accessible the authors could summarise the findings from studies conducted in Sub-Saharan African populations. For example, a table that orders studies by outcome/disease type and summarises key study parameters: methods (cohort/populations, LD reference panel, method) and results (variance explained) would be useful to readers.

When referring to predictive power being limited in African populations, additional detail as to what the AUC or equivalents are would be useful to contextualize the scores and provide comparisons to scores in non-African populations e.g. scores in EUR and AFR for a similar trait.

We suggest that the authors could also address the variability in transferability of scores not only between super-populations (eg. AFR and EUR) but also between SSA populations and the potential contributory role of environmental factors. Potential paper to do this includes Kamiza, A.B. et al. Transferability of genetic risk scores in African populations. ${ }^{1}$ 
One of the most recent useful advances in PRS development for ancestrally diverse populations is the PRS-CSx method. ${ }^{2}$ Although this new method was published after the date cut-off for the manuscript, a comment could be added in the Discussion.

\section{Minor comments:}

Introduction:

Table 1 is a comprehensive summary of PRS tools. How are these ordered? Given the authors have previously classified the methods into four groups (p3), it might be useful to use this information in structuring the table. In addition, what parameters/factors were used to determine whether an approach was user-friendly?

P3 "PRS analysis is used to predict an individual heritability by incorporating all selected SNPs." What do the authors mean here by the phrase 'individual heritability'? PRS methods that incorporate LD. In practice, when the markers are LD pruned...

PRS analysis on African populations:

This number represents about $4.553483 \%$ of total hits that - this number represents about $4.55 \%$ or $4.6 \%$ of total hits that

They observed that the predictive power of genetic risk scores was higher among African Americans ( $n=9139)$ and European Americans $(n=9594)$ relative to the sub-Saharan African populations $(n=5200)$.

- Consistency in number formatting: They observed that the predictive power of genetic risk scores was higher among African Americans $(n=9139)$ and European Americans $(n=9594)$ relative to the sub-Saharan African populations $(n=5200)$.

\section{References}

1. Kamiza AB, Toure SM, Vujkovic M, Machipisa T, et al.: Transferability of genetic risk scores in African populations.Nat Med. 2022; 28 (6): 1163-1166 PubMed Abstract | Publisher Full Text 2. Ruan Y, Lin YF, Feng YA, Chen CY, et al.: Improving polygenic prediction in ancestrally diverse populations.Nat Genet. 2022; 54 (5): 573-580 PubMed Abstract | Publisher Full Text

\section{Is the topic of the review discussed comprehensively in the context of the current literature?}

Yes

Are all factual statements correct and adequately supported by citations?

Yes

Is the review written in accessible language?

Yes

Are the conclusions drawn appropriate in the context of the current research literature? Yes

Competing Interests: Cathryn M. Lewis is a member of the SAB at Myriad Neuroscience 
Reviewer Expertise: Statistical Genetics, Genetic epidemiology

I confirm that I have read this submission and believe that I have an appropriate level of expertise to confirm that it is of an acceptable scientific standard, however I have significant reservations, as outlined above.

Author Response 17 Jan 2023

Ezekiel Adebiyi

Answers from the authors to the reviewer's comments

Authors would like to thank the reviewer for valuable comments and suggestions. Below are the answers for each reviewer's comments:

Major comments responses

1. To make the application of polygenic scores more accessible the authors could summarise the findings from studies conducted in Sub-Saharan African populations. For example, a table that orders studies by outcome/disease type and summarises key study parameters: methods (cohort/populations, LD reference panel, method) and results (variance explained) would be useful to readers.

Authors thank the reviewer for his suggestions. Authors think that this point is quite similar to a comment raised by reviewer 1 . Therefore, we address these two comments together by adding Table (4), pages 17-19.

2. When referring to predictive power being limited in African populations, additional detail as to what the AUC or equivalents are would be useful to contextualize the scores and provide comparisons to scores in non-African populations e.g. scores in EUR and AFR for a similar trait.

Authors thank the reviewer for his suggestions, and we agree that adding AUC as a method for evaluating PRS method will be useful for readers who are not familiar with machine learning and model evaluation. Therefore, we addressed this point by adding a text about AUC in the main manuscript.

3. We suggest that the authors could also address the variability in transferability of scores not only between super-populations (eg. AFR and EUR) but also between SSA populations and the potential contributory role of environmental factors. Potential paper to do this includes Kamiza, A.B. et al. Transferability of genetic risk scores in African populations

Authors accepted the reviewer's comment, we added the text below on page 19 of the revised version. We also added table 5 to give a summary of Transferability of PRS. in African populations.

"Previous studies suggested that PRS derived from individuals of African ancestry performed significantly better in sub-Saharan Africans than PRS derived from individuals of 
African Americans and Europeans and multiancestry (Duncan et al., 2019; Cavazos \& Witte, 20210; Martin et al., 2019; Johnson et al., 2015). However, PRS might differ across subSaharan Africans populations due to differences in contributory role of environmental and genetic factors. For instance, Kamiza et al\} reported that the differences in environmental and genetic factors play critical roles in transferability of PRS between the South African Zulu and individuals from Ugandan cohort Kamiza et al (2022), Table 5.. Finding from Kamiza et al\} noted that the poor performance of PRS across populations has implementation impact in preventative healthcare. Therefore, applying PPRS to different ethnic groups, even within within sub-Saharan Africa, may lead to inaccurate result. This further suggests the need for more efforts to optimize polygenic prediction in Africa. For instance, Choudhury et al (2022) demonstrated that PRS transferability among African can be improved by sample size of the African cohort studies."

\section{References}

Duncan, L., Shen, H., Gelaye, B. et al. Analysis of polygenic risk score usage and performance in diverse human populations. Nat Commun 10, 3328 (2019). https://doi.org/10.1038/s41467-019-11112-0

Cavazos, T. B., \& Witte, J. S. (2021). Inclusion of variants discovered from diverse populations improves polygenic risk score transferability. HGG advances, 2(1), 100017. https://doi.org/10.1016/j.xhgg.2020.100017

Martin, A. R., Kanai, M., Kamatani, Y., Okada, Y., Neale, B. M., \& Daly, M. J. (2019). Clinical use of current polygenic risk scores may exacerbate health disparities. Nature genetics, 51(4), 584-591. https://doi.org/10.1038/s41588-019-0379-x

Johnson, L., Zhu, J., Scott, E. R., \& Wineinger, N. E. (2015). An Examination of the Relationship between Lipid Levels and Associated Genetic Markers across Racial/Ethnic Populations in the Multi-Ethnic Study of Atherosclerosis. PloS one, 10(5), e0126361.

https://doi.org/10.1371/journal.pone.0126361

Kamiza AB, Toure SM, Vujkovic M, Machipisa T, Soremekun OS, Kintu C, Corpas M, Pirie F, Young E, Gill D, Sandhu MS, Kaleebu P, Nyirenda M, Motala AA, Chikowore T, Fatumo S. Transferability of genetic risk scores in African populations. Nat Med. 2022 Jun;28(6):11631166. doi: 10.1038/s41591-022-01835-x. Epub 2022 Jun 2. PMID: 35654908; PMCID: PMC9205766.

\section{One of the most recent useful advances in PRS development for ancestrally diverse populations is the PRS-CSx method.2 Although this new method was published after the date cut-off for the manuscript, a comment could be added in the Discussion.}

Authors thank the reviewer for the comment and we agree that PRS-CSx method is on the key method that can be used for the application of PRS across multi-ethnic group. We did not include because we submitted our review before publishing PRS-CSx method. However, we have include an overview of the PRS-CSx method in our reviewed manuscript, page 11. 
We also cited the article for those are interested to know more about its underlying algorithm.

\section{PRS-CSx method}

PRS-CSx method is proposed to improve the accuracy of the application of PRS across multiethnic populations by using posterior inference algorithm (Ruan et al., 2022; Ge et al., 2022). PRS-CSx combines GWAS summary files from different population to increase the accuracy of PRS. PRS-CSx estimates population-specific effect size by incorporating the populationspecific LD pattern, population-specific allele frequency information, and the information of shared continuous shrinkage prior across populations. For more details about the mathematical method underlying PRS-CSx, refer to Ruan et al., 2022).

\section{References}

Ruan, Y., Lin, YF., Feng, YC.A. et al. Improving polygenic prediction in ancestrally diverse populations. Nat Genet 54, 573-580 (2022). https://doi.org/10.1038/s41588-022-01054-7

Ge, T., Irvin, M. R., Patki, A., Srinivasasainagendra, V., Lin, Y. F., Tiwari, H. K., Armstrong, N. D., Benoit, B., Chen, C. Y., Choi, K. W., Cimino, J. J., Davis, B. H., Dikilitas, O., Etheridge, B., Feng, Y. A., Gainer, V., Huang, H., Jarvik, G. P., Kachulis, C., Kenny, E. E., ... Karlson, E. W. (2022). Development and validation of a trans-ancestry polygenic risk score for type 2 diabetes in diverse populations. Genome medicine, 14(1), 70. https://doi.org/10.1186/s13073022-01074-2

\section{Minor comments:}

Table 1 is a comprehensive summary of PRS tools. How are these ordered? Given the authors have previously classified the methods into four groups (p3), it might be useful to use this information in structuring the table. In addition, what parameters/factors were used to determine whether an approach was user-friendly?

The authors thank the reviewer for the comments. We determined whether an approach is user-friendly based on the installation process, the popularity of the methods among the users, the availability of an application manual (tutorial), the application user interface, and the number of options that should be considered and tuned by users. However, we have removed a column in the table (no.) that classifies the methods from the revised version based on four different approaches, such as (p3): clumping with thresholding $(C+T), p$-value thresholding, penalized regression, and Bayesian shrinkage, because some tools perform PRS analysis using more than one method. For instance, LDpred can perform PRS analysis either using clumping and thresholding $(\mathrm{C}+\mathrm{T})$ or the $\mathrm{p}$-value thresholding method.

We also cited et al . (2022), which performed a recent a comparison on some of these tools.

\section{References}

Wang Y, Tsuo K, Kanai M, Neale BM, Martin AR. Challenges and Opportunities for Developing More Generalizable Polygenic Risk Scores. Annu Rev Biomed Data Sci. 2022 Aug 10;5:293-320. doi: 10.1146/annurev-biodatasci-111721-074830. Epub 2022 May 16. PMID: 35576555 . 
P3 "PRS analysis is used to predict an individual heritability by incorporating all selected SNPs." What do the authors mean here by the phrase 'individual heritability'? PRS methods that incorporate LD. In practice, when the markers are LD pruned...

We mean by individual heritability here by the proportion of trait variance (phenotype) that is associated with genetic variants (genotype). We cited Privé et al (2020) \& Vilhjálmsson et al (2015) that provided more details of individual heritability that can be explained by the genetic variants.

\section{References}

Florian Privé, Julyan Arbel, Bjarni J Vilhjálmsson, LDpred2: better, faster, stronger, Bioinformatics, Volume 36, Issue 22-23, 1 December 2020, Pages 5424-5431, https://doi.org/10.1093/bioinformatics/btaa1029

Vilhjálmsson BJ, Yang J, Finucane HK, Gusev A, Lindström S, Ripke S, Genovese G, Loh PR, Bhatia G, Do R, Hayeck T, Won HH; Schizophrenia Working Group of the Psychiatric Genomics Consortium, Discovery, Biology, and Risk of Inherited Variants in Breast Cancer (DRIVE) study, Kathiresan S, Pato M, Pato C, Tamimi R, Stahl E, Zaitlen N, Pasaniuc B, Belbin G, Kenny EE, Schierup MH, De Jager P, Patsopoulos NA, McCarroll S, Daly M, Purcell S, Chasman D, Neale B, Goddard M, Visscher PM, Kraft P, Patterson N, Price AL. Modeling Linkage Disequilibrium Increases Accuracy of Polygenic Risk Scores. Am J Hum Genet. 2015 Oct 1;97(4):576-92. doi: 10.1016/j.ajhg.2015.09.001. PMID: 26430803; PMCID: PMC4596916.

PRS analysis on African populations: This number represents about $4.553483 \%$ of total hits that - this number represents about $4.55 \%$ or $4.6 \%$ of total hits that Authors thank the reviewer for this comment. We updated the Pubmed search terms that we used and formatted all numbers accordingly.

They observed that the predictive power of genetic risk scores was higher among African Americans ( $n=9139)$ and European Americans $(n=9594)$ relative to the sub-Saharan African populations $(n=5200)$. - Consistency in number formatting: They observed that the predictive power of genetic risk scores was higher among African Americans ( $n=9139)$ and European Americans ( $n=9594)$ relative to the sub-Saharan African populations $(n=5200)$.

Authors thank the reviewer for this comment. We formatted the style of all numbers accordingly.

Competing Interests: Authors declare no conflict of interest. 
(C) 2022 Zhao B. This is an open access peer review report distributed under the terms of the Creative Commons Attribution License, which permits unrestricted use, distribution, and reproduction in any medium, provided the original work is properly cited.

\section{Bingxin Zhao}

Department of Statistics, Purdue University, West Lafayette, IN, USA

This is an interesting paper on the review of PRS, with a focus on African populations. The research question is interesting and the writing is knowledgeable. I have the following suggestions that might improve the quality of this paper.

\section{Major:}

1. Although the title states that this paper focuses on African populations, I feel the current version of this paper is a bit too focused on general PRS research in all populations. I encourage the authors to include more details and examples of PRS applications in African populations, especially sub-Saharan African communities. Then, the paper may be more balanced.

For example, in Section "PRS analysis on African populations": The traits studied using PRS analysis in African populations include types 1 \& 2 diabetes mellitus, depression, ischemic stroke, schizophrenia, sarcoidosis, Alzheimer's disease, obesity, insomnia disorder, post-traumatic stress and cancer. Undermentioned are some selected PRS studies in sub-Saharan African populations.

The citations of these studies could be provided in the paper or a supplemental table. Then, the authors could provide a general overview of all the findings of these papers in the main text or supplemental note.

This is just one example, the authors could consider improve other sections of the paper to extend the discussions of African populations.

2. In the "The predictive power of PRS analysis" section, the authors could discuss and include a few more recent studies on the mathematical properties of PRS, such as https://onlinelibrary.wiley.com/doi/10.1111/biom.13466 and https://arxiv.org/abs/1911.10142. Particularly, https://arxiv.org/abs/1911.10142 studies the cross-population accuracy, which may fit the topic and discussion paper well.

\section{Minor:}

1. In the beginning of the "PRS methods that incorporate LD" section, the "W" in "When" should be lower case?

2. In the sentence "Feng \& Smoller 24presented the PRS-CS-auto method, a fully Bayesian approach that enables automatic learning of..." The citation (24) of Feng and Smoller is wrong?

3. "For instance, searching PubMed for PRS in African populations on August 21, 2021 (see Figure 1 and Box 1), only gave 8,843 hits." What is the result for sub-Saharan African?

4. "This number represents about $4.553483 \%$ of total hits that" $4.55 \%$ could be enough here. 


\section{References}

1. Zhao B, Zou F: On polygenic risk scores for complex traits prediction.Biometrics. 2021. PubMed Abstract | Publisher Full Text

Is the topic of the review discussed comprehensively in the context of the current literature?

Yes

Are all factual statements correct and adequately supported by citations? Yes

Is the review written in accessible language?

Yes

Are the conclusions drawn appropriate in the context of the current research literature? Yes

Competing Interests: No competing interests were disclosed.

Reviewer Expertise: genetics and statistics

I confirm that I have read this submission and believe that I have an appropriate level of expertise to confirm that it is of an acceptable scientific standard, however I have significant reservations, as outlined above.

Author Response 17 Jan 2023

Ezekiel Adebiyi

Answers from the authors to the reviewer's comments Authors would like to thank the reviewer for valuable comments and suggestions. Below are the answers for each reviewer's comments:

Our Response to the Major Comments

1. Although the title states that this paper focuses on African populations, I feel the current version of this paper is a bit too focused on general PRS research in all populations. I encourage the authors to include more details and examples of PRS applications in African populations, especially sub-Saharan African communities. Then, the paper may be more balanced.

For example, in Section "PRS analysis on African populations": The traits studied using PRS analysis in African populations include types 1 \& 2 diabetes mellitus, depression, ischemic stroke, schizophrenia, sarcoidosis, Alzheimer's disease, obesity, insomnia disorder, post-traumatic stress and cancer. Undermentioned are some selected PRS 
studies in sub-Saharan African populations.

The citations of these studies could be provided in the paper or a supplemental table. Then, the authors could provide a general overview of all the findings of these papers in the main text or supplemental note.

This is just one example, the authors could consider improve other sections of the paper to extend the discussions of African populations.

The Authors thank the reviewer for this major comment and we agree totally with the reviewer's point of view. Therefore, we have reviewed the content and also changed the title-heading "PRS Analysis on African Populations" to more specific title-header "PRS Analysis on Sub-Saharan African Populations", page 15. More so, we have cited the key articles and summarized the findings in the main text of the manuscript while we included additional table content, pages 17-19. The following text is provided for the revised version of the manuscript.

\section{PRS Analysis on Sub-Saharan African Populations}

The PRS Analysis on Sub-Saharan African populations is limited due to lack of enough GWAS studies on traits associated with Sub-Saharan African populations. For instance, searches on PubMed for PRS on Sub-Saharan African populations on December 23, 2022 (see Figure 1 and Box 1 ) results in only 5 hits (4 research articles and 1 review paper). These four research articles performed PRS analysis mainly on traits associated with cardiometabolic disease such as heart attack, type 2 diabetes, and stroke. Other contributing risk factors including body mass index (BMI), waist circumference (WC), hip circumference (HC), waist-to-hip ratio (WHR), systolic blood pressure (SBP), diastolic blood pressure (DBP), triglycerides (TG), total cholesterol (TC), low-density lipoprotein(LDL), high-density lipoprotein (HDL), fasting plasma glucose( FPG), and type 2 diabetes (T2D), low-density lipoprotein cholesterol (LDL-C), highdensity lipoprotein cholesterol (HDL-C), triglycerides (TGs) and total cholesterol (TC) (Ekoru et al., 2021; Kamiza et al., 2022; Choudhury et al., 2022; Meeks et al., 2022). More so, the variance detected for Sub-Saharan populations in these studies has been summarized in Table 4.

The general outcome of these five articles emphasize an urgent needs of GWAS research studies for Sub-Saharan African populations in order to continue to perform PRS analysis that would add more benefit to the use of PRS in precision medicine as well as an improved representation of multiple ethnic populations in GWAS to better reflect risk stratification, variabilities in genetic equitable, and translation of GRS in clinical setting . For instance, Ekoru et al (2021) demonstrated that several traits such as cardiometabolic have less predictive power of genetics risk score in Sub-Saharan Africans compared to others populations such as African Americans and European Americans. The less predictive power of cardiometabolic traits were as a result of underrepresented African populations based on GWAS data in the current reference genomes. However, Kamiza et al. (2022) studies showed an increase in PRS performance on lipid traits (such as, LDL-C) with dataset from Sub-

Saharan populations, European, and multi-ancestry. Other lipid traits include HDL-C, TGs and TC. PRSs performance varies significantly even among the sub-Saharan African populations. This variation on PRS performance occurs due to variations on Africa 
population-specific genetic structure, such as minor allele frequencies and the populationspecific associated environmental factors.

It is worth reporting that there are several PRS studies that have been done using African populations. However, these studies are not restricted to sub-Saharan Africa's populations because the 1000 genomes reference panel data include samples from Africa populations. In 2020, Hayat and her colleagues investigated the genetic associations between serum low LDL-cholesterol levels and selected genetic variants (Hayat et al., 2020). Using 1000 genomes data from the African populations, they selected four genes for their investigation (LDLR, APOB, PCSK, and LDLRAP1). They performed genotyping of 19 SNPs using 1000 participants in the Human Heredity and Health in Africa (H3Africa) AWI-Gen Collaborative Center (Africa, Wits-IN-DEPTH Partnership for GENomic studies). Although they used a limited number of variants, the outcome showed a significant association of these SNPS with lower LDL-C levels in sub-Saharan Africans.

In 2020, Cavazos and Witte proposed the inclusion of variants discovered from various populations to improve PRS transferability to diverse populations (Cavazos and Witte, 2020). They used both simulated data for the Yoruba group of the sub-Saharan African and European populations. They tested their findings on real data consisting of diabetes-free training samples of European ancestry $(n=123,665)$ and African descent $(n=7564)$. They evaluated the performance of PRS analysis using genotype and phenotype data for a test (predictive) data set of European ancestry $(n=394472)$ individuals of African origin from the UK Biobank $(n=5886)$. Based on their findings, they concluded that incorporating variants selected from the European population will limit the accuracy of PRS values in nonEuropeans populations including African communities. Also, they commented on the need for diverse GWAS data to improve PRS accuracy across populations.

In 2017, Marquez-Luna et al. (2017) proposed a multi-ethnic PRS analysis to improve risk prediction in diverse populations including African communities. To overcome the lack of enough training data for the African populations, the authors combined the training data from European samples and training data from the target population. We did not include their study because they did not state whether they used sub-Saharan African communities. This further highlights the challenge of performing PRS analysis in sub-Saharan African populations as a result of insufficient training data.

In 2017, Vassos et al. (2017) examined PRS values in a group of individuals with first-episode psychosis (Vassos et al., 2017). For the control data set, they combined African-European ( $n=$ 70) and a sample of sub-Saharan African ancestries $(n=828)$. Their finding showed that PRS value was more potent in Europeans, i.e. 9.4\% discriminative ability, than in Africans, i.e. only $1.1 \%$ discriminative ability in Africans.

\section{References}

Badianyama, M., Mpanya, D., Adamu, U., Sigauke, F., Nel, S. and Tsabedze, N., 2022. New biomarkers and their potential role in heart failure treatment optimisation-An African 
perspective. Journal of Cardiovascular Development and Disease, 9(10), p.335.

Ekoru K, Adeyemo AA, Chen G, Doumatey AP, Zhou J, Bentley AR, Shriner D, Rotimi CN. Genetic risk scores for cardiometabolic traits in sub-Saharan African populations. Int J Epidemiol. 2021 Aug 30;50(4):1283-1296. doi: 10.1093/ije/dyab046. PMID: 33729508; PMCID: PMC8407873.

Kamiza AB, Toure SM, Vujkovic M, Machipisa T, Soremekun OS, Kintu C, Corpas M, Pirie F, Young E, Gill D, Sandhu MS, Kaleebu P, Nyirenda M, Motala AA, Chikowore T, Fatumo S. Transferability of genetic risk scores in African populations. Nat Med. 2022 Jun;28(6):11631166. doi: 10.1038/s41591-022-01835-x. Epub 2022 Jun 2. PMID: 35654908; PMCID: PMC9205766.

Choudhury A, Brandenburg JT, Chikowore T, Sengupta D, Boua PR, Crowther NJ, Agongo G, Asiki G, Gómez-Olivé FX, Kisiangani I, Maimela E, Masemola-Maphutha M, Micklesfield LK, Nonterah EA, Norris SA, Sorgho H, Tinto H, Tollman S, Graham SE, Willer CJ; AWI-Gen study; H3Africa Consortium, Hazelhurst S, Ramsay M. Meta-analysis of sub-Saharan African studies provides insights into genetic architecture of lipid traits. Nat Commun. 2022 May 11;13(1):2578. doi: 10.1038/s41467-022-30098-w. Erratum in: Nat Commun. 2022 Aug 2;13(1):4474. PMID: 35546142; PMCID: PMC9095599.

Meeks KAC, Bentley AR, Doumatey AP, Adeyemo AA, Rotimi CN. Mendelian randomization study reveals a causal relationship between adiponectin and LDL cholesterol in Africans. Sci Rep. 2022 Nov 8;12(1):18955. doi: 10.1038/s41598-022-21922-w. PMID: 36347891; PMCID: PMC9643497.

2. In the "The predictive power of PRS analysis" section, the authors could discuss and include a few more recent studies on the mathematical properties of PRS, such as https://onlinelibrary.wiley.com/doi/10.1111/biom.13466 and https://arxiv.org/abs/1911.10142. Particularly, https://arxiv.org/abs/1911.10142 studies the cross-population accuracy, which may fit the topic and discussion paper well.

Authors thank the reviewer for his valuable suggestion. We added the text below to the manuscript, page 13, to improve it.

"Zhao \& Zou (2022) showed in their study that PRS predictivity can be improved based on SNPs selection. The process of SNPs selection depends on the genetic architecture, i.e, causal variants, and the sample size of the training data set. To select a set of SNPs that provide the optimal PRS prediction, the sample size of the training data set should be much larger than the number of potential causal variants. That is, performing PRS where the ratio of causal variants and sample size is large results in poor PRS prediction due failure in causal variants separations. Therfores, in the case of the ratio of causal variants to the sample size is large, i.e, small sample size is the training data set, Zhao \& Zou recommended to include a large number of variants to get higher PRS prediction power. Moreover, Zhao \& Zou recommended to include independent uncorrelated variants to 
improve PRS predictivity. Moverover, Zhao et al. (2022) demonstrated that accounting for correlation between causal variants, i.e, LD, will improve PRS predictivity and accuracy for heterogeneous populations.

Furthermore, the performance of the PRS mathematical model can be assessed by evaluating the model's output using machine learning techniques, including area under the curve (AUC) of the receiver operating characteristic (ROC) (Janssens et al., 2007; Igo et al., 2019). The ROC can be visualized by plotting true positive rate against false positive rate for model's thresholds. Janssens et al. (2007) recommend using a model that provides AUC $>0.75$ for PRS clinical utility, ie, screening of individuals who are at risk. Igo et al. (2019) has suggested using the proportion of trait variability explained by one or more variants as an indicator for PRS predictivity, for more details refer to Janssens et al. (2007).

Igo, R. P., Jr, Kinzy, T. G., \& Cooke Bailey, J. N. (2019). Genetic Risk Scores. Current protocols in human genetics, 104(1), e95. https://doi.org/10.1002/cphg.95

Janssens, A. C., Moonesinghe, R., Yang, Q., Steyerberg, E. W., van Duijn, C. M., \& Khoury, M. J. (2007). The impact of genotype frequencies on the clinical validity of genomic profiling for predicting common chronic diseases. Genetics in Medicine, 9, 528-535. doi: 10.1097/GIM.0b013e31812eece0.

Zhao, B., \& Zou, F. (2022). On polygenic risk scores for complex traits prediction. Biometrics, 78(2), 499-511. https://doi.org/10.1111/biom.13466

Zhao, B., Zou, F., \& Zhu, H. (2022). Cross-trait prediction accuracy of summary statistics in genome-wide association studies. Biometrics, 10.1111/biom.13661. Advance online publication. https://doi.org/10.1111/biom.13661

\section{Authors responses to the Minor comment}

1. In the beginning of the "PRS methods that incorporate LD" section, the "W" in "When" should be lower case?

Authors thank the reviewers for the suggestions, and we applied the correction accordingly.

\section{In the sentence "Feng \& Smoller 24presented the PRS-CS-auto method, a fully} Bayesian approach that enables automatic learning of..." The citation (24) of Feng and Smoller is wrong?

Authors thank the reviewers for the suggestions, and we applied the correction accordingly. We cited it as $\mathrm{Ge}$ et a as given is the correct citation below

Ge T, Chen C-Y, Ni Y, Feng Y-CA, Smoller JW. Polygenic prediction via Bayesian regression and continuous shrinkage priors. Nat Commun. 2019;10(1):1776.

3. "For instance, searching PubMed for PRS in African populations on August 21, 2021 (see Figure 1 and Box 1), only gave 8,843 hits." What is the result for sub-Saharan African?

Authors thank the reviewers for the suggestions, we updated our search terms and we have added the recent results, including PubMed hits for sub-Saharan African in Figure 1. 
4. "This number represents about $4.553483 \%$ of total hits that" $4.55 \%$ could be enough here.

We thank the reviewer, such small number demonstrate the lack of enough PRS studies on African populations.

Competing Interests: Authors declare no conflict of interest.

The benefits of publishing with F1000Research:

- Your article is published within days, with no editorial bias

- You can publish traditional articles, null/negative results, case reports, data notes and more

- The peer review process is transparent and collaborative

- Your article is indexed in PubMed after passing peer review

- Dedicated customer support at every stage

For pre-submission enquiries, contact research@f1000.com 